\title{
Lattice Design Study for the HEB to Collider Transfer Lines
}

F. Wang, K. Brown, J. McGill, and D. Johnson

\author{
Superconducting Super Collider Laboratory* \\ 2550 Beckleymeade Ave. \\ Dallas, TX 75237
}

May 1993

"Operated by the Universities Research Association, Inc., for the U.S. Department of Energy under Contract No. DE-AC35-89ER40486. 


\section{CONTENTS}

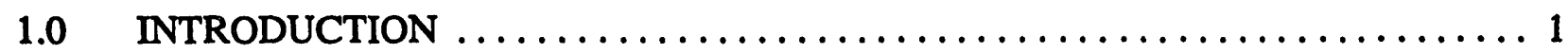

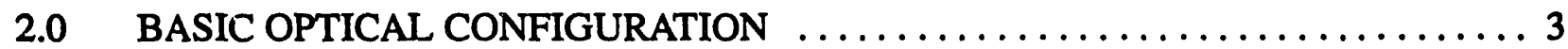

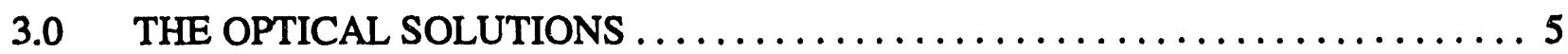

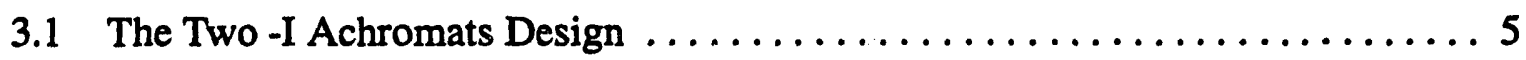

3.2 Two Achromats Approach with Resistive Magnets (Old Warm) . . . . . . . 8

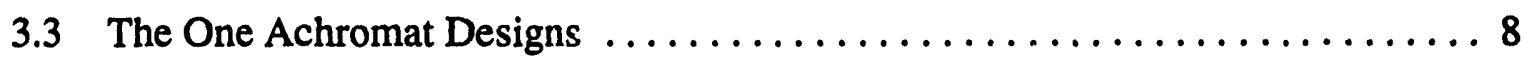

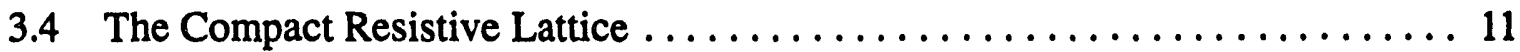

4.0 COMPARISON OF OPTICAL FLEXIBILITY $\ldots \ldots \ldots \ldots \ldots \ldots \ldots \ldots \ldots \ldots$

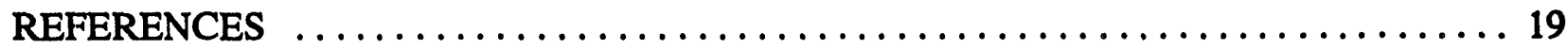

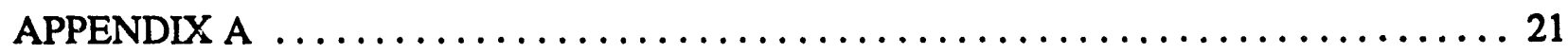

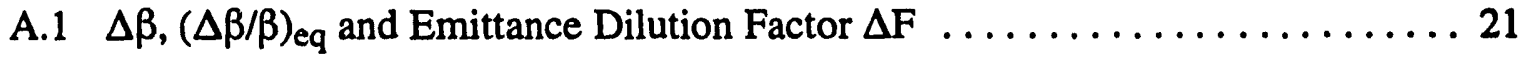

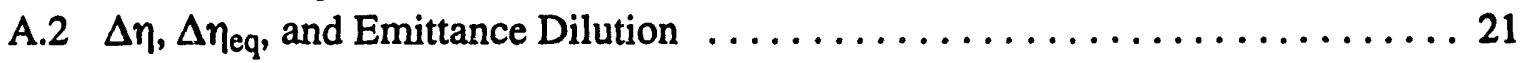

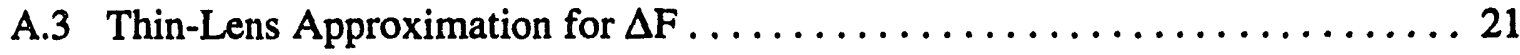

APPENDIX B: SENSITIVITY TO QUADRUPOLE GRADIENT ERRORS . ...... 23 


\section{FIGURES}

1. Layout of the HEB-to-Collider Transfer Lines $\ldots \ldots \ldots \ldots \ldots \ldots \ldots \ldots \ldots$

2. Lattice Functions of the Compact Resistive Solution $\ldots \ldots \ldots \ldots \ldots \ldots \ldots$

3. Lattice Designs for the HEB-to-Collider Transfer Lines $\ldots \ldots \ldots \ldots \ldots \ldots \ldots$

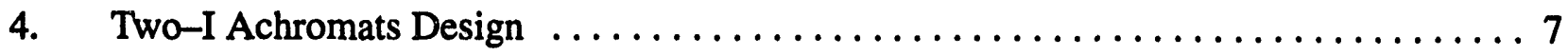

5. Dispersion Vectors of Compact Lattice $\ldots \ldots \ldots \ldots \ldots \ldots \ldots \ldots \ldots \ldots \ldots \ldots$

6. Compact Resistive Lattice with Different Independent Quadrupole PS Numbers . . . 12

7. Equal Matching for Old Warm and Compact Lattice $\ldots \ldots \ldots \ldots \ldots \ldots$

8. Optical Flexibility for $-\mathrm{I}$, Old Warm, and Hybrid Solutions $\ldots \ldots \ldots \ldots \ldots \ldots \ldots$

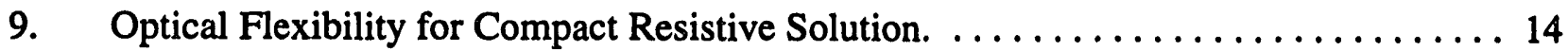

10. Optical Flexibility Study (Compact Resistive Lattice) $\ldots \ldots \ldots \ldots \ldots \ldots$

B-1. Sensitivity to Gradient Errors (Compact Resistive Lattice) $\ldots \ldots \ldots \ldots \ldots \ldots$

\section{TABLES}

1. Optics Flexibility Study for Compact Resistive Lattice $\ldots \ldots \ldots \ldots \ldots$

B-1. Comparision of Sensitivitiy to Quadrupole Gradient Errors . . . . . . . . . . 24 


\subsection{INTRODUCTION}

The High Energy Booster (HEB) ring is the last booster of the 20-TeV Superconducting Super Collider (SSC). The 2-TeV beam transfer lines between these two giant accelerator complexes should meet all geometrical and optical matching conditions while having sufficient tuning flexibility. The lattice design of these lines also has to be concerned with the huge energy each HEB extracted batch carries (6.55MJ). A compact lattice design with all magnets being resistive was adopted for its optical flexibility, operational reliability, and cost saving.

The HEB west long straight section (HWLS) is directly over the two collider west utility straight sections (WUSS). The verical separation between HEB and the bottom collider is $14 \mathrm{~m}$, which is determined by radiation safety requirements. The elevation separation between the two colliders is $0.9 \mathrm{~m}$.

There are two beam lines to transfer both the clockwise (CW) and counterclockwise (CCW) extracted HEB beams to top collider and bottom collider, respectively. Geometric boundaries of these lines are fixed by the global machine layout. The two transfer lines are in the same vertical plane, which is about $18 \mathrm{~mm}$ inside of either the HEB or the Colliders' west long straight sections. There are no horizontal bending magnets in these lines; the small horizontal shift of the transfer plane is generated by horizontal kickers. The length of each beam line is approximatly $640 \mathrm{~m}$. To transfer a $2-\mathrm{TeV}$ proton beam, this length is relatively short.

The Site-Specific Conceptual Design Report (SCDR) presented a superconducting approach for this transfer system ${ }^{1}$ that closely fits the present global layout. The design used several types of Fermi National Accelerator Laboratory (FNAL) superconducting magnets. Lattice structures of the two lines were similar, which is important to maintain uniformity in operation. This criterion has been applied to all subsequent designs. Concerns of superconducting magnet quenching problems were reflected in the SCDR by way of a suggestion to consider using resistive magnets throughout the beamline.

In 1991, a resistive magnet solution ${ }^{2}$ with $14-\mathrm{m}$ vertical separation from HEB to bottom collider had been worked out. However, the use of iron-dominated magnets, limited to $1.8 \mathrm{~T}$, basically filled approximatly half the length of the transfer line with dipoles. The consequent restrictions on the placement of quadrupoles resulted in an irregular beta function and limited tuning flexibility. But it was realized that building any superconducting magnets other than HEB or collider superconducting magnets would be extremely costly. Further study of optics with the possibility of using existing HEB or collider superconducting magnets to improve the lattice was required. ${ }^{3}$

An intensive study of lattice design was performed in 1992, resulting in several different designs: a lattice with two -I achromats; ${ }^{4}$ a hybrid solution ${ }^{5}$ using superconducting and resistive magnets; and a compact resistive magnet solution. ${ }^{6}$ This systematic approach has given us a clear scope of what we can do for optics with considerations of technical and budget constraints.

The compact resistive solution has been adopted because its cost is lower and it has fewer problems of physical interference. Since all magnets are warm, one can consider implementation of a collimator system to protect collider elements from HEB extract kicker misfire. The optics of this lattice is compact and flexible. Figure 1 shows the layout of this design, and Figure 2 depicts various optical functions.

The design study here in many ways is an effort to solve inter-accelerator transfer line optical problems dealing with insufficient phase advance and strict matching requirements. 


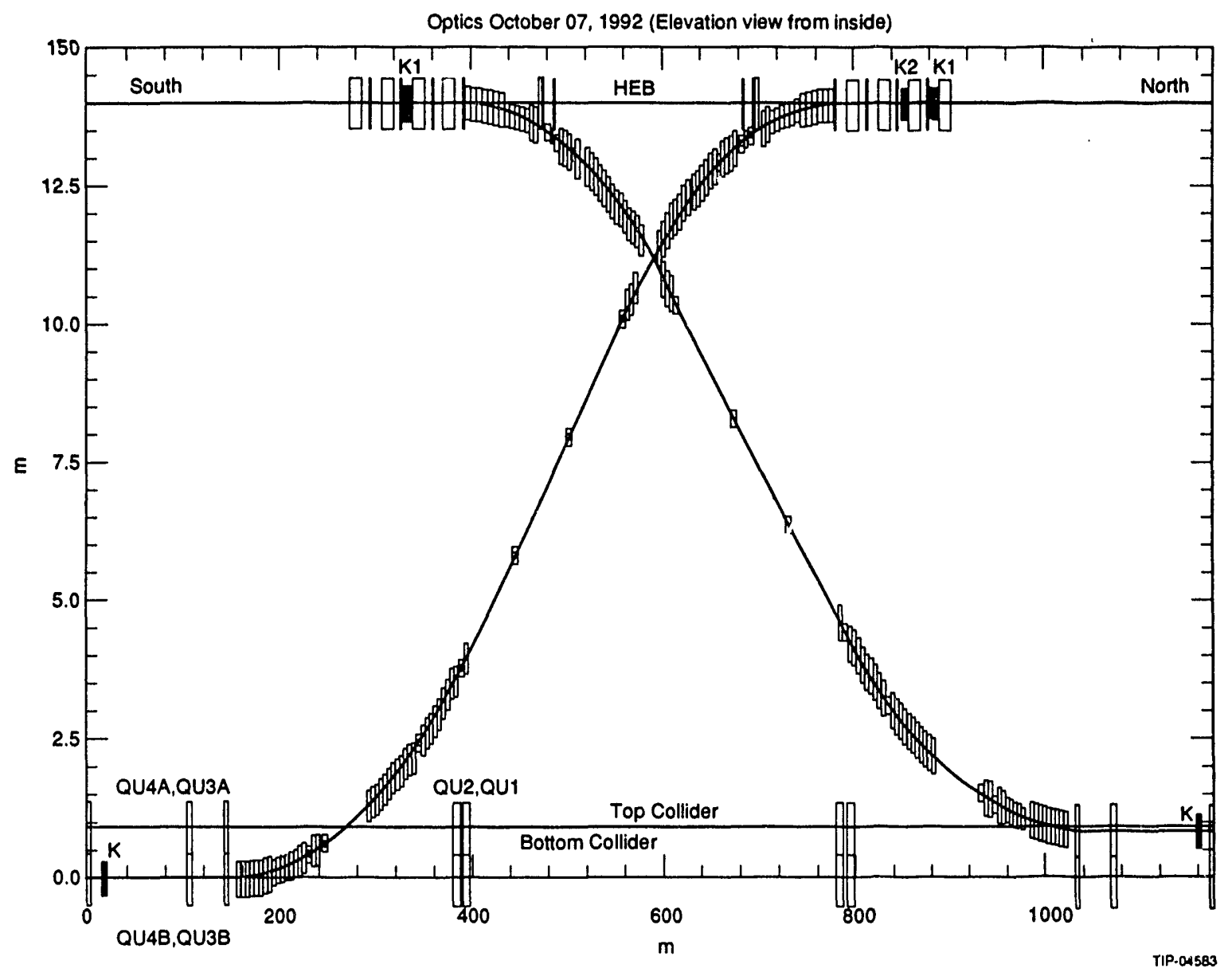

Figure 1. Layout of the HEB-to-Collider Transfer Lines. 

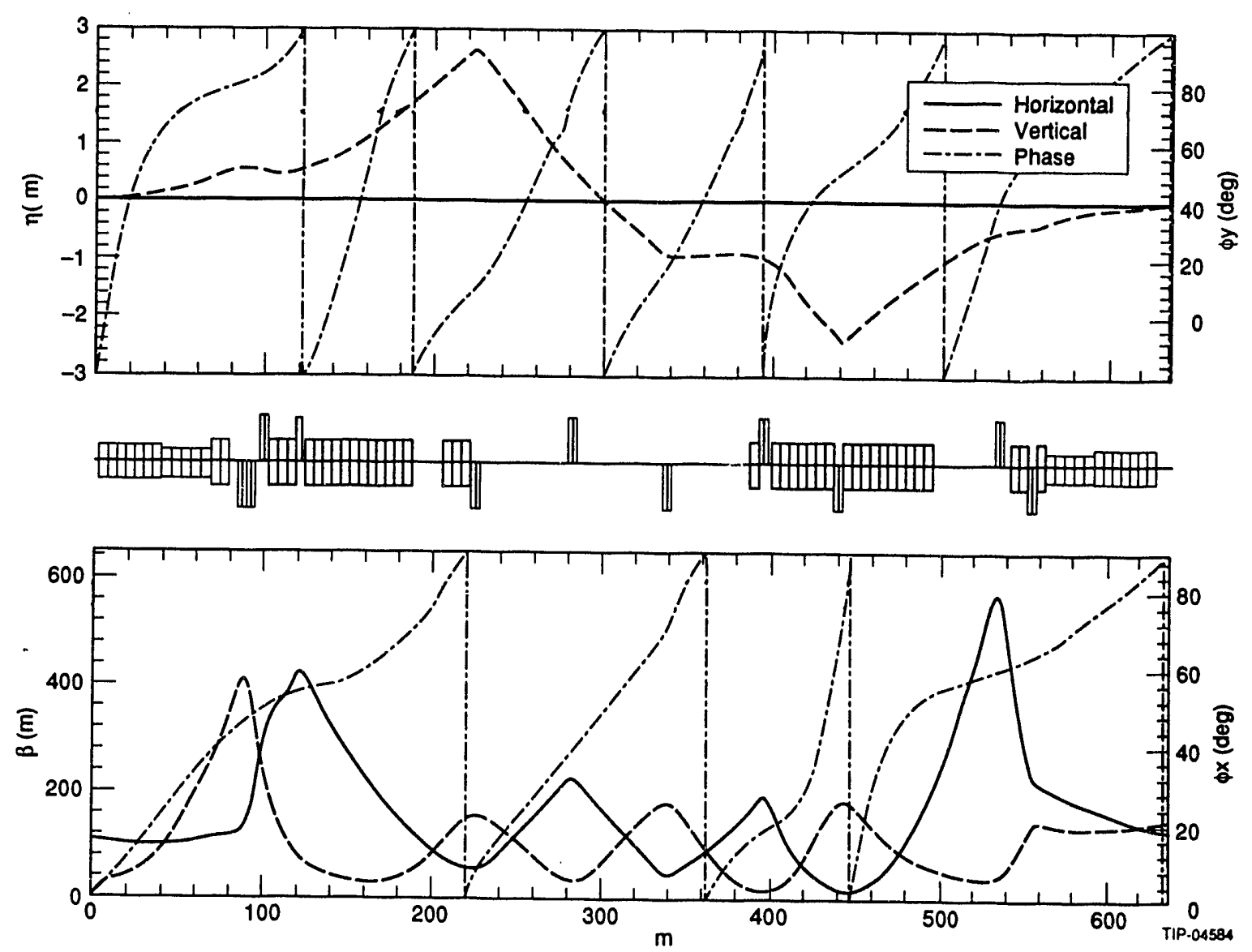

Figure 2. Lattice Functions of the Compact Resistive Solution.

\subsection{BASIC OPTICAL CONFIGURATION ${ }^{7}$}

The basic optical design goals of these lines are (a) centroid matching, i.e., closed orbit matching; (b) $\beta$ matching; and (c) dispersion function matching.

To meet the layout requirements, these beam lines contain only vertical bending magnets. Each transfer line is a parallel beam axis shift system. One group of bending magnets bends the beam down and the other group of dipoles bends the beam up.

The HEB west long straight section and collider utility straight sections are dispersion-free regions. Therefore the transfer line should be an achromatic system.

The beam line lattices may fall into the following three categories by dealing with $\eta$ and $\beta$ matching differently:

1. $\eta$ matching first, $\beta$ matching second. This type of lattice typically has two achromats on both ends and a Twiss matching section between. Orthogonal trim of $\beta$ and $\eta$ functions may be attainable. Control of $\beta$ and $\eta$ functions orthogonally will make beam line tuning easy and operation simple, which is one of the design goals if achievable. When making achromats, proper control of $\beta$ amplitudes is always a concern. A two -I transfer lattice was made in the belief that it was the best strategy for this approach. 
2. $\beta$ matching first, $\eta$ matching second. In this case, one places Twiss matching sections at each end, matching the HEB and collider $\beta$ functions into a periodic structure in the middle, say FODO. Then by placing the two dipole groups, with opposite bending directions properly, one can match coordinates and close the dispersion vector diagram (Section 3.3).

Most of our designs proceeded in this way, as dipoles have little effect on Twiss parameter matching. However the game to "place dipoles properly" is not straight forward, even when a dispersion vector diagram has been drawn, because the vector is a combination of bending angle and local $\beta$ amplitude. Also the positions available for dipoles and quadrupoles are badly constrained by interference problems with HEB, Colliders, and even the beamlines thernselves.

An example of such an approach is the SCDR design, which matches both ends to a FODO structure between. Then by adjusting the phase advance in the FODO cells and introducing a small dogleg in the center, the vertical dispersion is cancelled. However due to insufficient phase advance in the FODO structure, adjustment of $\eta$ and $\beta$ matching has to be mixed to some extent.

3. Mixed $\eta$ and $\beta$ matching. The two prececiug lattice designs applied to these lines all need superconducting dipoles and quadrupoles to generate sufficient bending angle and betatron phase advance.

Mixed $\eta$ and $\beta$ matching must be implemented in our hybrid or resistive magnet solutions because there is insufficient phase advance to separate the two functions. This makes the problem difficult to solve in a systematic way, and orthogonal tuning for these lines is not possible.

Three designs of this kind have been tested:

The first was a warm (all resistive magnets) solution (Oct. 1991, the "old warm lattice"), which attempted to work out two achromats but did not succeed. The 13 quadrupoles had to be tuned simultaneously to match all conditions.

The second was a hybrid solution, which used one HEB superconducting dipole and one HEB quadrupole at each end of the beam lines to help control $\beta$ amplitudes. When working on this solution, we tried to match Twiss parameters on both ends first.

The third design (compact resistive) was an evolution of the hybrid solution above. Realizing that with resistive magnets, this transfer length is too short to separate $\eta$ and $\beta$ matching, we decided to make a simple piece of lattice to match both $\eta$ and $\beta$ directly from HEB to Collider. However some kind of $\beta$ amplitude control is still needeci on each end. Instead of making "Optical Insults" (highly irregular $\beta$ matching section) as in case 2, here two quadrupoles were used at each end to reduce maximum $\beta$ amplitude and to present reasonable $\beta$ functions to start a periodical oscillation.

The following data may give a feeling of what phase advance can be expected for a warm solution:

- $67 \mathrm{~m}$ is needed for either extraction or injection septum and C-magnets (field $\sim 1.1 \mathrm{~T}$ ) array to shift the beam $32 \mathrm{~cm}$ away from the ring orbit. This is the space required to install a high-field dipole magnet. ${ }^{8}$ The remaining length of transfer line is $\sim 500 \mathrm{~m}$. This is the space available for matching the optics and geometry of the beam.

- Possible phase advance with limited Q gradient strength, and reasonable $\beta$ amplitude. For $90^{\circ}$ FODO (thin lens approximation) the $2 \pi$ structure length $^{9}$ is:

$$
8 L \approx \frac{8}{3.4} \beta_{\max }=2.35 \beta_{\max }
$$


where $L$ is the distance between adjacent quadrupole centers, and $\beta_{\max }$ is the maximum beta amplitude of the structure. The quadrupole focal length is:

$$
f=0.7 L=\frac{B \varrho}{G l},
$$

where $G$ is the field gradient, $l$ is the effective length, and $B Q$ is the beam rigidity.

Assuming quadrupole length $l=5 \mathrm{~m}$ and gradient $G=32 \mathrm{~T} / \mathrm{m}$, for a $2-\mathrm{TeV}$ beam one obtains $f=41.7 \mathrm{~m}$. Choosing a reasonable number for $\beta_{\max }$, say $200 \mathrm{~m}$, one has a structure length of $470 \mathrm{~m}$. This means that the phase advance with warm quads can be no larger than $\sim 2 \pi+$ phase advances in extraction and injection septum and $\mathrm{C}$-magnets.

As the two bending groups bend the beam in opposite directions, to cancel dispersions the "bend centers" of the two groups should be $2 n \pi$ apart. As stated in Section 3.3, the "bend center" here is a combined gravity of bending angle and $\beta$ amplitude in phase space. However, in our case they are close to geometric bending centers. Bending angle of all septa and C-magnets is about $1 / 3$ of total bending angle needed. Therefore geometrical bending centers will be out of septa and $\mathrm{C}$-magnets and closer to the center.

Obviously with resistive magnets, the possible choice to closely cancel dispersions by positioning dipoles is to make a $2 \pi$ phase advance between these two "bend centers."

\subsection{THE OPTICAL SOLUTIONS}

Five lattice designs are discussed here. Figure 3 is a compact plot of their optical functions.

\subsection{The Two -I Achromats Design}

In this design there is a-I achromat on each end of the transfer line, and a $\beta$-matching section in the center part (see Figure 4). The design was constrained to use HEB-type dipole or quadrupoles if they have to be superconducting.

Each -I achromat consists of three HEB-type dipoles and an array of septa and C-magnets. One of these HEB dipoles (the middle of the three) is separately powered to couple the septum and C-magnets array at $\pi$ phase advance in order to cancel dispersion generated by each other. The other two HEB dipoles are powered in series, also $\pi$-phase apart. By changing the bending field of these two dipoles, it is possible to steer the beam while not introducing dispersion. There are five quadrupoles to constitute two $90^{\circ} \mathrm{FODO}$ cells in each of these two achromats. One of the quadrupoles is split.

The minimum FODO cell length ( $\pi / 2$ phase advance) is about the length of the septum and C-magnet array, since the independently powered dipole has to be placed between two quadrupoles to make its center $\pi$-phase advance from the center of the septum and C-magnet array. Septum and C-magnets' array length is decided by space interference limits and magnet field limits for the septum and C-magnet, not for any optical reason. In our case, the $90^{\circ}$ FODO cell length is $\sim 87 \mathrm{~m}$ (septum and C-magnet length $\sim 70 \mathrm{~m}$ ).

A major difficulty for this design is the control of peak $\beta$-amplitudes in the -I achromats. The natural peak $\beta$ of a FODO structure is scale-to-cell length, see Eq. (1). As we cannot further reduce cell length to reduce peak $\beta$, one of the quadrupoles has to be split, and the other quadrupoles have to be powered separately to allow trimming their gradients individually. This is a setback to the ideal of separating dispersion-matching and $\beta$-matching. The other way to lower $\beta$-peak is to reduce phase advance in the FODO cell, say, by going to a $60^{\circ}$ FODO. However, we failed to do that due to insufficient length of the line. 
(a)
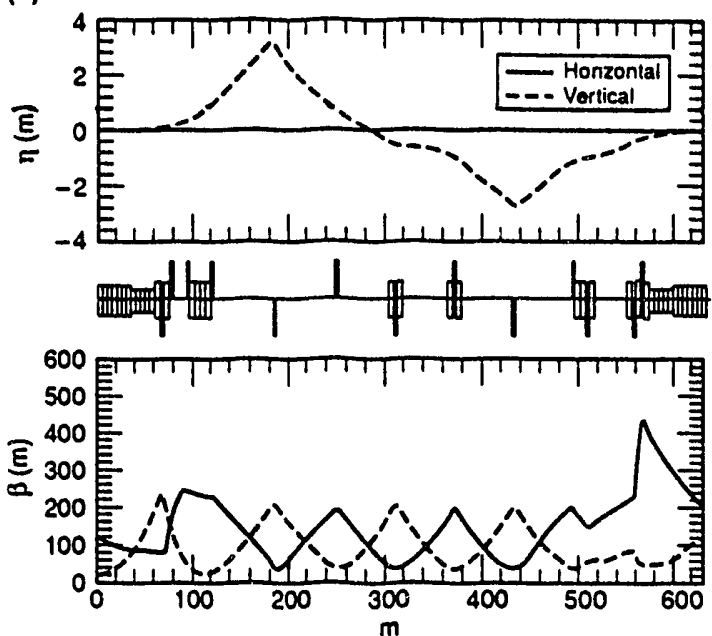

(c)
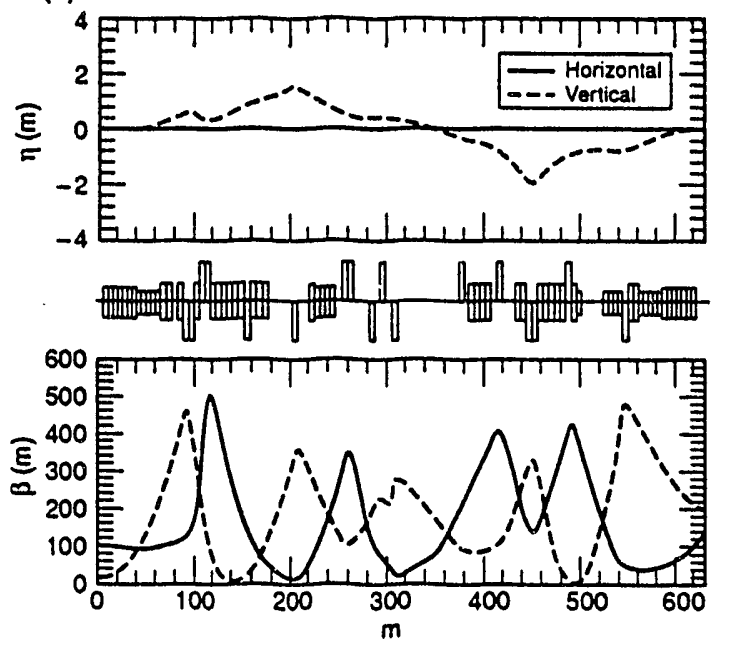

(b)
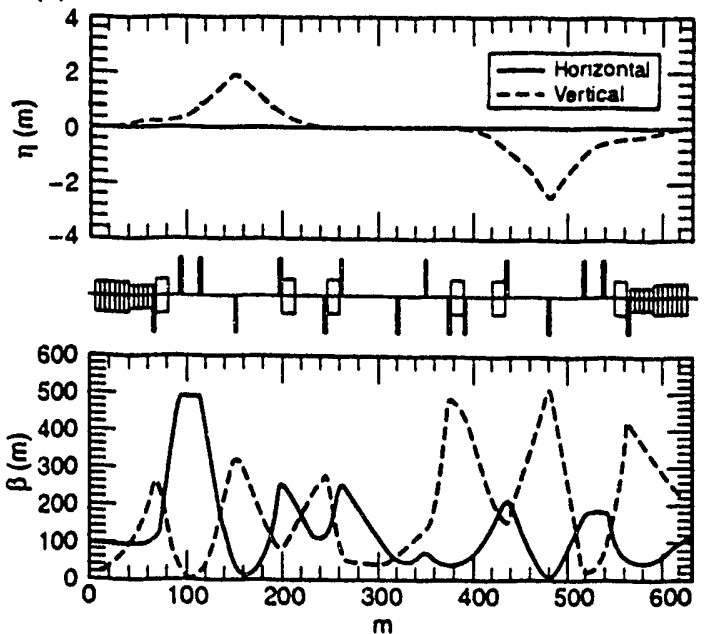

(d)
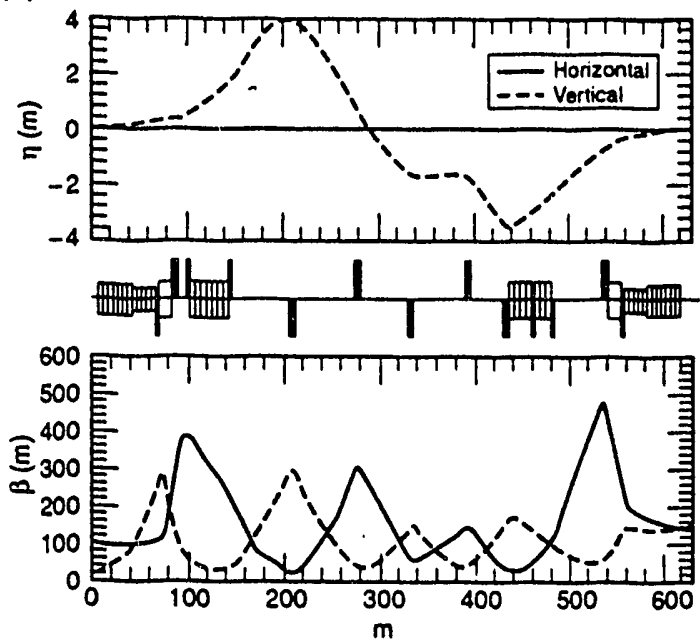

(e)
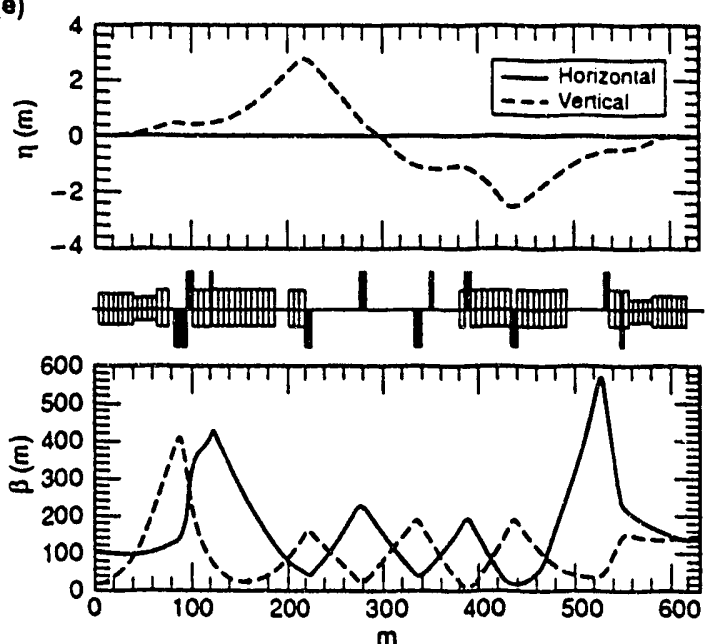

Figure 3. Lattice Designs for the HEB-to-Collider Transfer Lines. (a) SCDR Design, (b) Two -1 Achromats, (c) Resistive One (Old Warm), (d) Hybrid Lattlce, (e) Compact Resistive. 
(a)

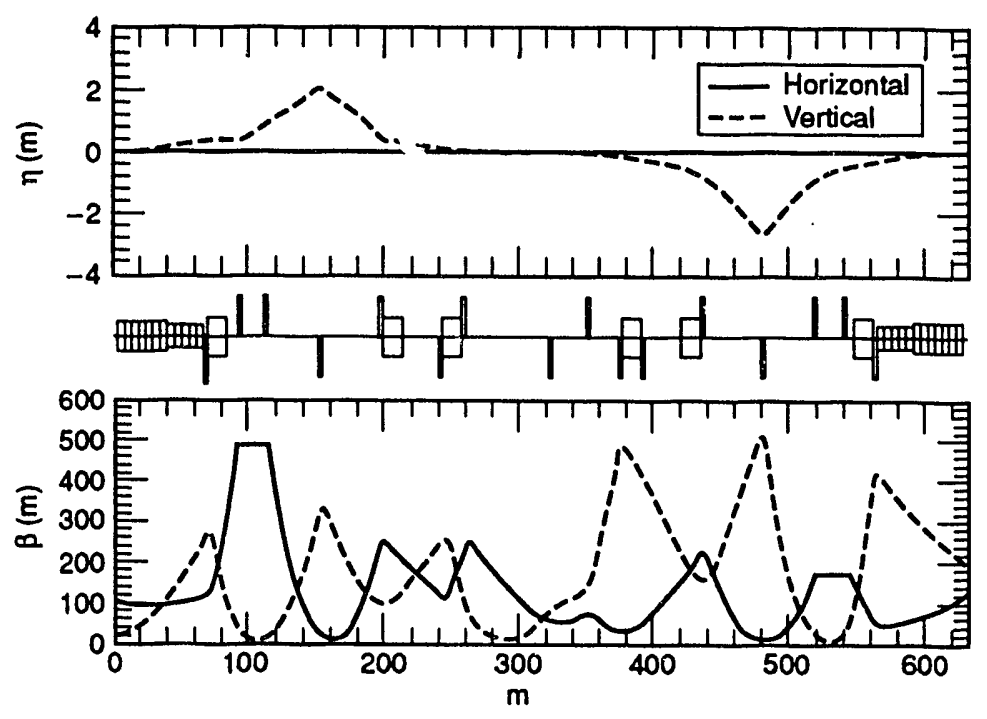

(b)
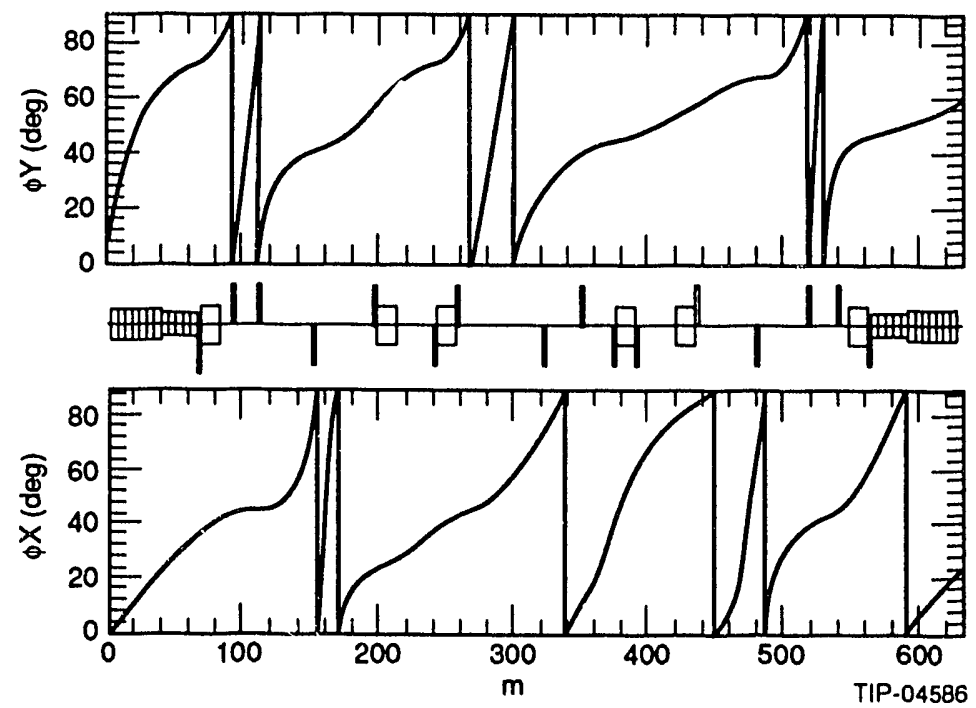

Figure 4. Two $\dashv$ Achromats Design.

The remaining length in the center part for $\beta$-matching is $\sim 140 \mathrm{~m}$. To match $\beta$ in a very confined space is also a difficult task, though we solved it in this case. In cases that $\beta$-matching cannot be accomplished in this section, it will extend to achromats, thus mixing the two matching again.

In Section 4.0, we will see that these space limits will spoil the orthogonal control and degrade optical flexibility to this ideal design.

The maximum dispersion in a two-achromat system is small $(<\sim 2 \mathrm{~m})$, compared to a one-achromat lattice. But this is not a sensitive issue in these designs, due to the small fractional momentum spread $\left(\sim 10^{-4}\right)$ of the $2-\mathrm{TeV}$ HEB beam. 


\subsection{Two Achromats Approach with Resistive Magnets (Old Warm)}

This was the first approach to make a resistive solution for these lines. The intention was to make two achromats. However, with the low bending magnet field limit $(\sim 1.8 \mathrm{~T})$, the resistive dipoles just spread to the center if one tries to balance the bending centers to cancel $\eta$ on both ends. Even then it was still not possible to succeed with two achromats and to keep $\beta$-amplitude under control. That is, the maximum $\beta$-amplitude should be comparable to collider maximum $\beta$, so that the transfer line will not be a restricting aperture in normal operation. Also the effects of magnet errors are sensitive to large $\beta$ s (e.g., see Appendix B). Efforts had been made to confront space interferences all along the line while maintaining a reasonable $\beta$-amplitude, $<\sim 500 \mathrm{~m}$. To match the full range of initial conditions, all quadrupole gradients had to be varied simultaneously.

Optically the solution has an average large and irregular $\beta$-distribution (Figure 3(b)), which brings into doubt its optical flexibility.

\subsection{The One Achromat Designs}

As mentioned earlier, the process of this approach usually is to match $\beta$ first, because bends have little effect on $\beta$-matching. However, to reduce the total bending power, the bending center should be pushed as close as possible to both ends. In general, the lattice should have a $\beta$-matching section on each end to match machine optics and to provide a FODO array between.

The maximum $\beta$-amplitude in the HEB regular cell is about $100 \mathrm{~m}$, and it is $-300 \mathrm{~m}$ in the collider. A reasonable value for maximum $\beta$ of the line FODO is about $200 \mathrm{~m}$, so that a length of $470 \mathrm{~m}$ is required for $2 \pi$-phase advance using $90^{\circ} \mathrm{FODO}$ cells. This is just the approximate length between the ends of the septum and C-magnet arrays. We saw in Section 2.0 that the integral gradient of a quadrupole for such a $90^{\circ} \mathrm{FODO}$ is about $160 \mathrm{~T}$. This is about half the strength of a superconducting HEB cell quadrupole $(-290 \mathrm{~T})$. So if we want more phase advance, say $4 \pi$, it will require very strong quadrupoles $(\sim 320 \mathrm{~T})$. This may be possible for a design with superconducting magnets, but will be very costly!

Next let's clear the meaning of positioning the "bending centers" $2 \pi$ away and further examine the requirements of achromaticity. The general transfer matrix between position 1 and position 2 in a transfer system is ${ }^{9}$

$$
R=\left[\begin{array}{cc}
\sqrt{\frac{\beta_{2}}{\beta_{1}}\left(\cos \Delta \psi+\alpha_{1} \sin \Delta \psi\right.} & \sqrt{\beta_{1} \beta_{2}} \sin \Delta \psi \\
\frac{\left(1+\alpha_{1} \alpha_{2}\right) \sin (\Delta \psi)+\left(\alpha_{2}-\alpha_{1}\right) \cos \Delta \psi}{\sqrt{\beta_{1} \beta_{2}}} & \sqrt{\frac{\beta_{1}}{\beta_{2}}}\left(\cos \Delta \psi-\alpha_{2} \sin \Delta \psi\right)
\end{array}\right]
$$

where $\Delta \psi$ is the phase shift between position 1 and 2 ,

If $2 \mathrm{n} \pi$ shift is imposed,

$$
R=\left[\begin{array}{cc}
\sqrt{\frac{\beta_{2}}{\beta_{1}}} & 0 \\
\frac{\left(\alpha_{2}-\alpha_{1}\right)}{\sqrt{\beta_{1} \beta_{2}}} & \sqrt{\frac{\beta_{1}}{\beta_{2}}}
\end{array}\right] .
$$

Equations (3) and (4) are the transfer matrices for the transverse coordinates $x$ and $x^{\prime}$. In a bend-free region, the dispersion vector will have the same transfer matrices. 
Now we assume that the bending groups can be represented by two point-bending centers and that they are $2 \pi$-phase apart. The first bend generates $\eta_{1}^{\prime}$ (which is approximately equal to the bending angle, say $\theta_{b 1}$ ) at position 1 , and $\eta_{1}=0$. The transfer space between the two bending centers is bend-free, so by Eq. (4) we have $\eta_{2}=0, \eta_{2}^{\prime}=\sqrt{\beta_{1} / \beta_{2}} \eta^{\prime}$, at position 2 .

To cancel this dispersion the second bend should have a bending angle $\theta_{b 2}=\sqrt{\beta_{2} / \beta_{1}} \theta_{b 1}$. In case of equal bending angles, it requires $\beta_{1}=\beta_{2}$. If the matching conditions are different at the two ends, both the phase advance and $\beta_{1}=\beta_{2}$ conditions would have to be modified.

Practically, the bending magnets are mixed with focussing quadrupoles, and in the strict sense " $2 \pi$ away bending centers" are not merely geometric centers of bending angles but combined centers of bending angle and $\beta$-amplitude in phase space. However, the above bending-center mode is a good initiation of how to place these magnets. For more precise descriptions, we start from the standard linear equation of transverse motion:

$$
X^{\prime \prime}+K(s) X=\frac{\Delta p}{p} \cdot \frac{1}{\mathrm{Q}(s)},
$$

where $X$ is the transverse coordinate; $s$, the longitudinal coordinate; $\Delta p / p$, the fractional momentum deviation; and $\mathrm{Q}(s)$ the radius of curvature at $s$.

In linear approximation, the transverse motion can be separated into two parts: a displaced trajectory of off-energy particles $\left(\mathrm{X}_{\eta}\right)$, and the free betatron oscillation about this trajectory $\left(\mathrm{X}_{\beta}\right)$. The trajectory of an off-energy particle $X_{\eta}=\eta \cdot \frac{\Delta p}{p}$ is the unique particular solution of Eq. (5) with the driving term $\frac{\Delta p}{p} \cdot \frac{1}{\mathrm{Q}}$.

In a bend-free region ( $1 / \varrho=0)$, from Eq. (5) the behavior of $X_{\eta}$ is similar to a free betatron oscillation. Therefore we can write the off-energy trajectory in a normalized phase space as:

$$
X_{\eta}^{2}+\left(\beta X_{\eta}^{\prime}+\alpha X_{\eta}\right)^{2}=\beta \varepsilon .
$$

The emittance $\varepsilon$ is an invariant determined by momentum offset $\Delta p / p$ and by the initial dispersion function. Using the relation $X_{\eta}=\eta \cdot \frac{\Delta p}{p}$ and $X_{\eta}{ }^{\prime}=\eta^{\prime} \cdot \frac{\Delta p}{p}$, one can further write a normalized phase-space expression for the dispersion function as

$$
\left(\frac{\eta}{\sqrt{\beta}}\right)^{2}+\left(\eta^{\prime} \sqrt{\beta}+\frac{\alpha}{\sqrt{\beta}} \eta\right)^{2}=\varepsilon_{\eta} .
$$

Here $\varepsilon_{\eta}$ is an invariant as well.

We can take the dispersion as a vector in phase space. The two orthogonal components of such a normalized dispersion vector are

$$
\frac{\eta}{\sqrt{\beta}} \text { and } \eta^{\prime} \sqrt{\beta}+\frac{\alpha}{\sqrt{\beta}} \eta \text {. }
$$

For a system consisting of many bending magnets, the resulting off-energy trajectory is the linear sum of those generated by individual bending magnets. In case of an achromatic system, the vector sum of these individual dispersion vectors should be zero (see Figure 5(a)). 
(a)

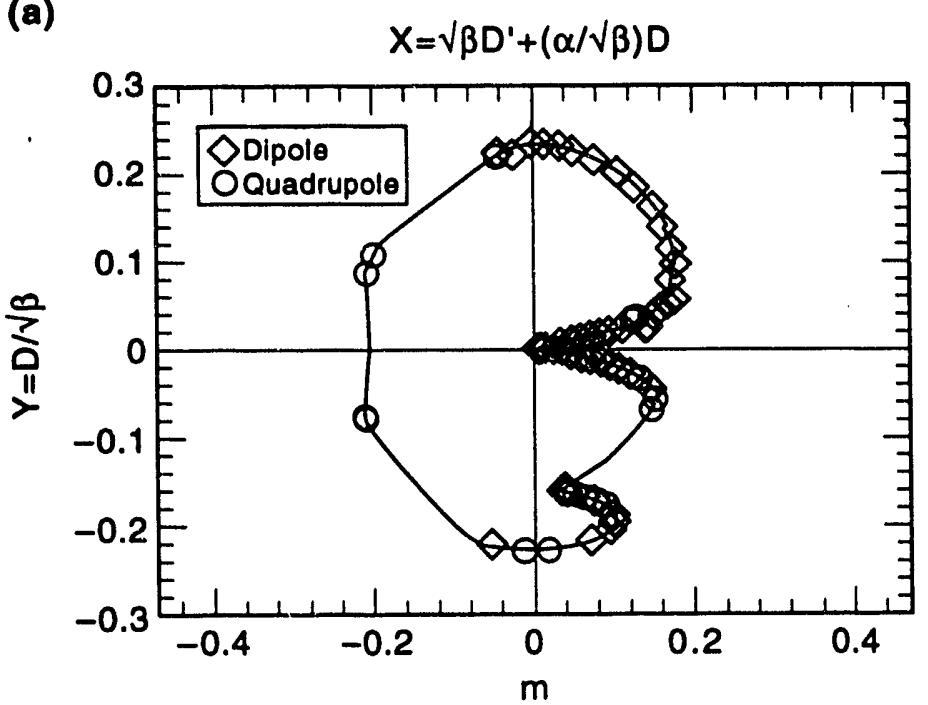

(b)

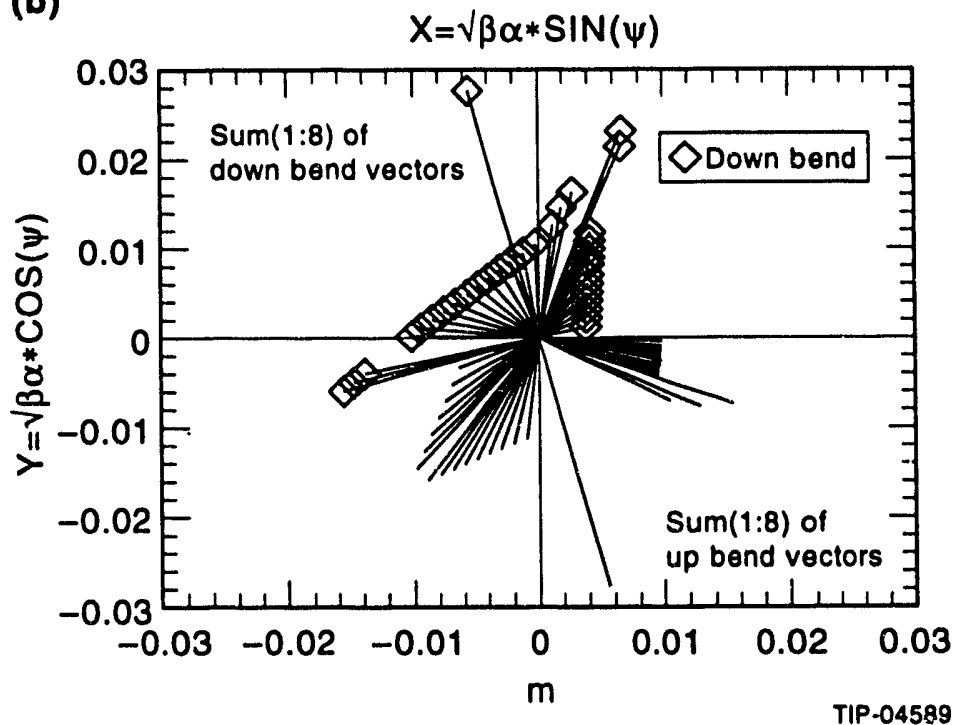

Figure 5. Dispersion Vectors of Compact Lattice. (a) FLOQUET Coordinates for the Dispersion Vector. (b) Dispersion Vectors by Dipoles.

An explicit way to describe the effect of each bend is to draw the normalized dispersion vector generated by each bend separately. Consider a single bend, with a small bending angle of $\theta_{b}$. The normalized dispersion vector that it generates is $\left(0, \sqrt{\beta} \theta_{b}\right)$. The normalized dispersion yector at a subsequent point can be derived from Eq. (3), and Eq.(7) becomes

$$
\left(\sqrt{\beta} \alpha_{b} \cos \psi\right)^{2}+\left(\sqrt{\beta} \alpha_{b} \sin \psi\right)^{2}=\varepsilon_{\eta}
$$

where $\beta$ is always the beta amplitude at the bend position and $\psi$ is the betatron phase advance from the bend to the point concerned.

Now starting from the beginning of the system, we can draw the dispersion vectors along with their phase angle in normalized phase space.This is shown in Figure 5(b) for the compact resistive solution. For an 
achromatic system the sum of all vectors should be null. A dispersion vector diagram may be illustrative, but not very instructive as to how one should proceed.

The SCDR design matches $\beta$ first and makes the FODO between (Figure 3(a)). The two opposite-direction bend groups have the same bending angle value, and the bend centers of the two are 360-deg phase apart. However the $\beta$ functions are dissimilar at these centers, as the line is matched into two rings with dissimilar $\beta$ functions. Also the bends are mixed with quadrupoles. So the cancellation of dispersion cannot be complete. Two mure variable parameters are provided: one is the phase shift in the FODO, and the other is a dogleg bending structure in the middle of the line. Further trimming of dispersion was not a issue to be addressed at that time. However orthogonal control of $\beta$ and $\eta$ in operation is not likely to be the case.

$I_{i}$ the systematic study of possible lattice designs, a hybrid solution was presented (Figure 3(d)). Constrained by using the fixed type of superconducting dipoles and quadrupoles, this design has only half the FODO cell of the SDCR design. The design process is again to match $\beta$ first; witl $\beta$-matching sections at both ends, match $\eta$ second. Usually $\eta$-matching is done in the middle section by varying quadrupoles (including those inside the bending groups) and even by moving dipole positions somewhat. Iterations are needed to match dispersion while keeping the $\beta$-oscillation periodic in the center. This is beneficial to tuning flexibility (see Section 4.0).

The cost of the all-superconducting solution (two -I design) is expected to be high because of the superconducting current bus, leads, etc. Also the possibility of implementing collimators in the line is dim. The hybrid design trades orthogonal control of $\eta$ and $\beta$ for money saving and the possibility of installing collimators. However to solve spatial conflicts with the collider and HEB, special cryostats still have to be developed in both ends. They are costly and would add technical complications in this troublesome region. So another pursuit to resistive lattice was pushed.

\subsection{The Compact Resistive Lattice}

The major change in this design is to give up trying to match on the two ends and to make a one-piece $\beta$ and $\eta$ - matching lattice.

It is easy to count how many variables are needed to match the HEB to the Collider :irectly: two for $\eta$ and four for Twiss parameters. Practically, one quadrupole on each side is added to have some preliminary control of $\beta$-amplitudes, which are the extensions of the $\beta$-oscillation in the straight sections of the rings.

To cancel dispersion more naturally, one has to pay attention to the $2 \pi$-phase shift between the two major bending centers. On the one hand, we want to push bends to both ends to save total bending power; on the other hand, we distribute some of the bending power of each bending group toward the center to balance the group bend center position.

The final matching of six parameters in most cases is accomplished by varying gradients of the "six quadrupoles" in the middle of the line. However, the two quadrupoles-one at each end-play important roles in shaping the $\beta$-oscillation wave forms in the center part (Figure 6 (a)). Iterations usually are carried out to make $\beta$-oscillations in the middle part closer to a periodical form.

The present compact resistive solution has a maximum $\beta$-amplitude $\sim 600 \mathrm{~m}$, and a fine pseudo-periodical structure in the middle. Ten quadrupoles and nine independent power supplies are used to further improve the $\beta$-oscillation waveform (Figure $6(\mathrm{~b})$ ).

The evolution of these solutions, in fact, is not totally based on the same boundary conditions. The hybrid and compact resistive solution fit the same collider straight section lattice, while the old warm solution and the -I solution fit the earlier collider lattice. 
(a)
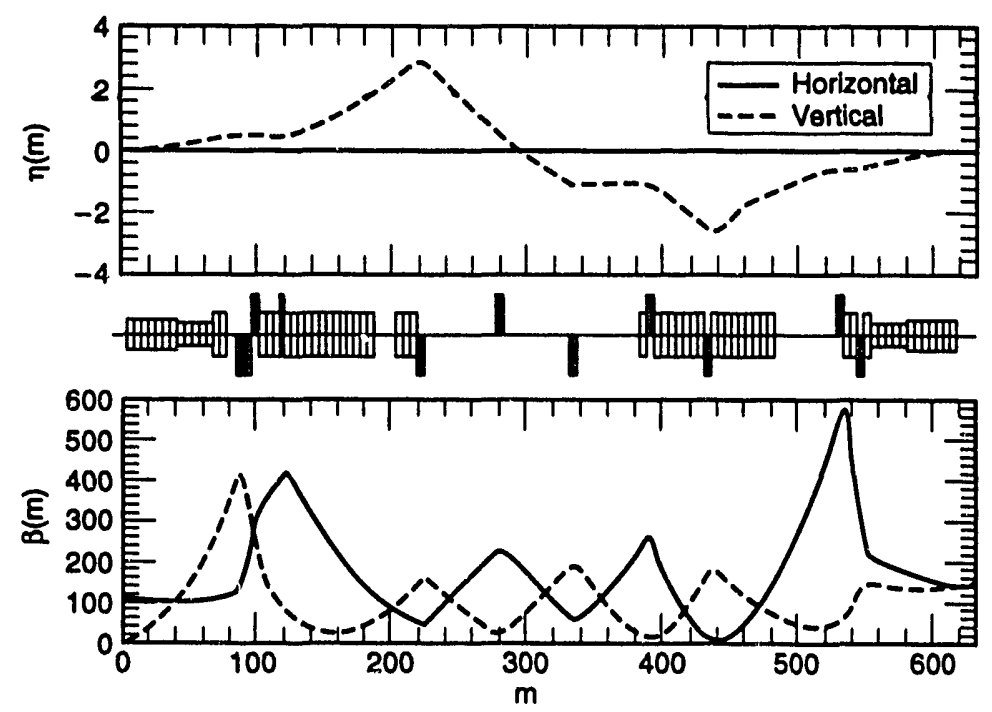

(b)

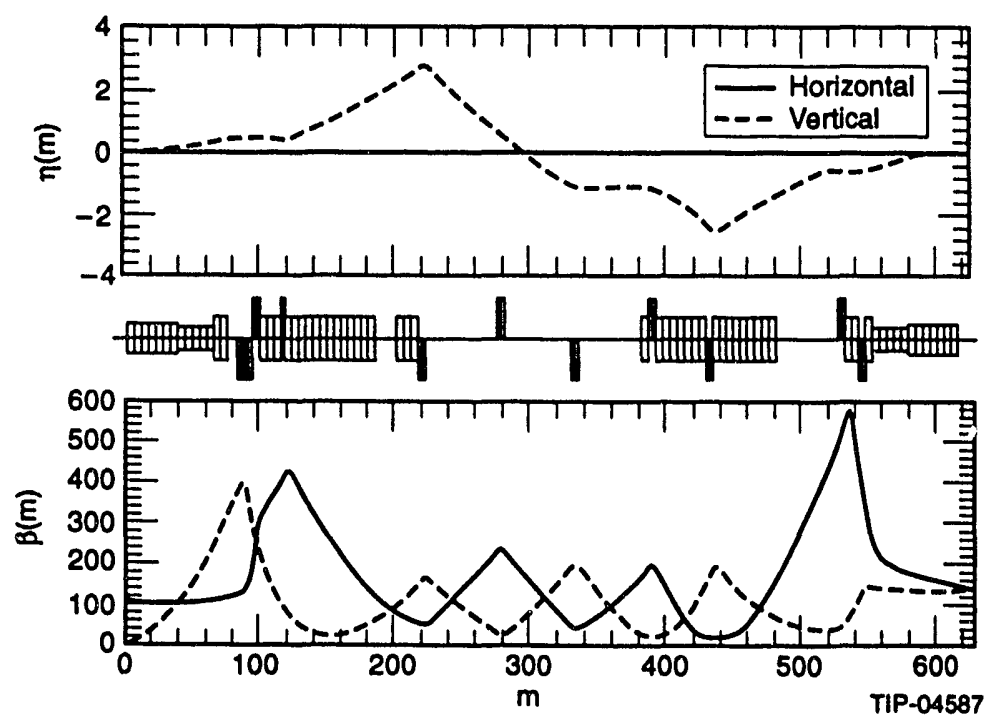

Flgure 6. Compact Resistive Lattice with Different Independent Quadrupole PS Numbers.

(a) Elght Quadrupole PS. (b) Nine Quadrupole PS.

Boundary conditions will definitely make a difference in matching or shaping $\beta$-oscillations; however the one-piece compact resistive layout does work to some extent. Figures 7(a) and (b) give an example with the same old boundary conditions for old warm solution and for the compact resistive lattice. Without any revision (rnoving magnet positions), the second one, which is designed to fit the new boundary conditions, fits the old matching conditions easily and improves the $\beta$-oscillation waveform. The merit of the compact resistive lattice shown in this example will be further examined in Section 4.0.

We now have arrived at a solution that has no orthogonal $\beta$ - and $\eta$ - control. Therefore, we must ask, what is the tuning flexibility? 
(a)
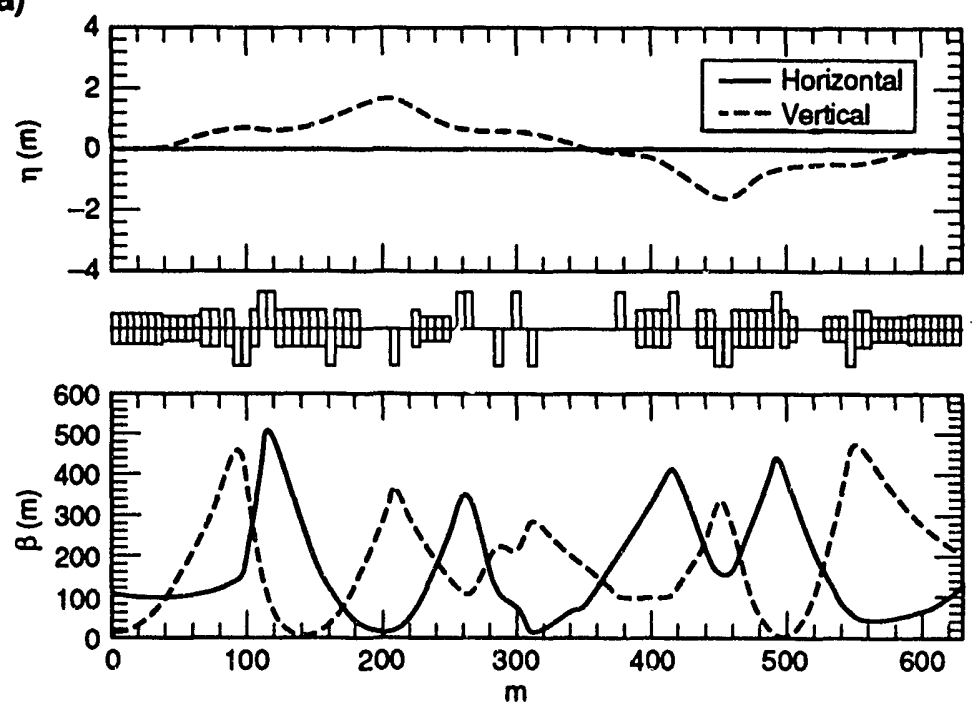

(b)
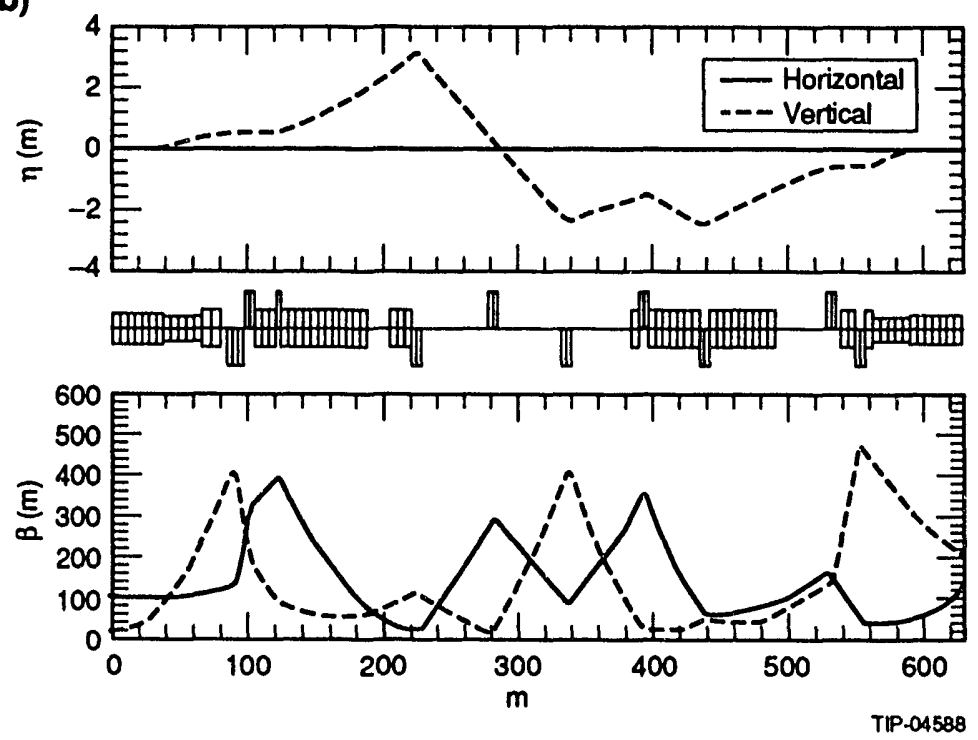

Figure 7. Equal Matching for Old Warm and Compact Lattlce. (a) First Warm Solution. (b) With Compact Structure.

\subsection{COMPARISON OF OPTICAL FLEXIBILITY}

In real machine operation, the matching conditions on both the HEB and collider sides may change to what may be good for the machine tuning. It is ideal to fit these new conditions without moving quadrupoles around in beam lines. In our case, the tolerances for mismatching are very small to prevent emittance dilution in colliders. In addition, the lattice structures are not regular, and in most designs $\beta$ - and $\eta$-control are not orthogonal.

The optical or tuning flexibility of the beam line may be defined as the matching range of HEB and Collider operating points that the beam line can accommodate. The criterion set for matching is the maximum allowed emittance dilution in the following machine (here, collider rings) when a perfect match is not possible. 
The constraints for beamline tuning are (1) quadrupole gradient strength limit, a technical limit, and (2) maximum betatron amplitude along the line, a consideration from beamline aperture and error sensitivity requirements. Figures 8 and 9 are graphical descriptions of the above statement.

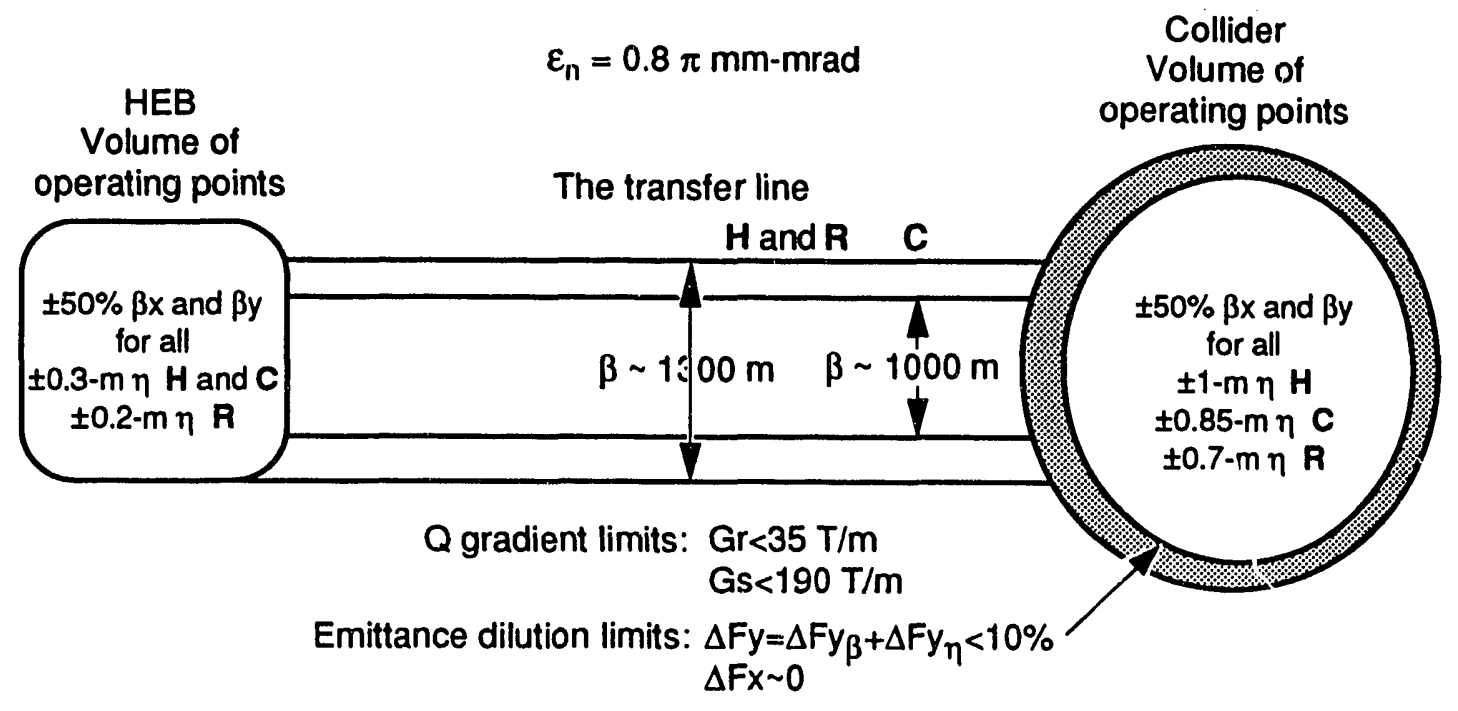

H: Hybrid solution, July 17, 1992 (new WUSS lattice July 6, 1992)

C: Cold solution, May 19, 1992 (-I, two Achromats)

R: Old warm solution, Oct. 24, 1991

TIP-04590

Figure 8. Optical Flexibility for -l, Old Warm, and Hybrid Solutions.

HEB

$\varepsilon_{\mathrm{n}}=0.8 \pi \mathrm{mm}-\mathrm{mrad}$

Collider

Volume of operating points

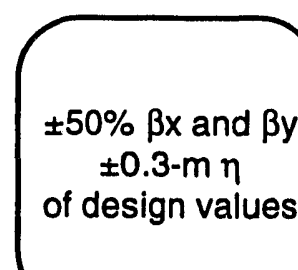

The transfer line

\begin{tabular}{|c|c|}
\hline The transfer line & \\
\hline Matching & $\begin{array}{l}E \\
8 \\
8 \\
0 \\
v \\
n\end{array}$ \\
\hline
\end{tabular}

Q gradient limits: $G<40 \mathrm{~T} / \mathrm{m}$

Volume of operating points 
Using common sense, one would ask how much change from the designed values of $\beta$ or $\eta$ in either the HEB or Collider straight sections can be made and the line still fit.

We have to link changes on $\beta$ - or $\eta$-values to emittance dilution properly (Appendix A). The allowed maximum fractional emittance dilution is $10 \%$, according to general requirements in the SCDR (for comparison purposes here only). This roughly corresponds to one transverse direction $+50 \% \beta$-mismatch, or to 1-m $\eta$-mismatch at the collider side.

Results if several fitting calculations for the $-\mathrm{I}$, hybrid, old warm, and compact resistive designs are depicted in Figures 8 and 9. To make a fair comparison, fitting was made by whatever means to deal with mathematical problems: quadrupoles can be grouped in different ways or in different fitting order. Fitting criteria for $\eta$ or $\beta$ may be different to obtain minimum emittance dilution value. All fittings were done with "TRANSPORT."

An amazing result is that the one-piece compact resistive solution can always make a perfect match in all matching conditions, while the others may result in some degree of dilution under some of these conditions. It is believed that the pseudo-periodical structure helps to accommodate a wide range of matching conditions by accommodating a wide range of $\beta$-amplitudes in the center part.

Table 1 and Figure 10 give an example of fitting for the compact resistive lattice. Figure 10(a) shows that when a $0.3-\mathrm{m} \eta$ is initiated in the HEB side, the result $\eta$ at the collider side is $-0.8 \mathrm{~m}$. In the worst case if a $+1-\mathrm{m}$ residual $\eta$ is required at the collider side, the line has to tune itself up to fit it. This is shown Figure $10(\mathrm{~b})$. The $\beta$-oscillation waveform is changed, and the pseudo-periodical structure allows the $\beta$-amplitude sufficient margin to expand in the middle to adjust the phase shift to meet the matching requirements. A more difficult situation is depicted in Figures $10(\mathrm{c})$ and $10(\mathrm{~d})$, where both $\eta$ and $\beta$ are required to match to controversial conditions. Table 1 lists the details of $\eta$ and $\beta$, dilution, quadrupole gradients before and after fitting. In case 4 (Figure 10(d)), one quadrupole field gradient is tuned up to $40 \mathrm{~T} / \mathrm{m}$, a $27 \%$ increase over design value. 
TABLE 1. OPTICS FLEXIBILITY STUDY FOR COMPACT RESISTIVE LATTICE.

\begin{tabular}{|c|c|c|c|c|c|c|c|c|c|c|}
\hline $\begin{array}{l}\text { The Design Parametor } \\
\text { HEB extraction } \\
\text { Collider Injection }\end{array}$ & $\begin{array}{c}8 x \\
110.2045 \\
139.0880\end{array}$ & $\begin{array}{c}\text { By } \\
18.8725 \\
154.8894\end{array}$ & $\begin{array}{c}A x \\
0.3108 \\
0.2396\end{array}$ & $\begin{array}{c}\text { AV } \\
-2.0215 \\
-0.3000\end{array}$ & $\begin{array}{c}G x \\
0.0100 \\
0.0078\end{array}$ & $\begin{array}{c}G v \\
0.0530 \\
0.0070\end{array}$ & $\begin{array}{l}\text { Elav } \\
0.0000 \\
0.0000\end{array}$ & $\begin{array}{l}\text { Eia'v } \\
0.0000 \\
0.0000\end{array}$ & Sigma(p) & $\begin{array}{c}\text { Emittance/(BC } \\
\text { (m Rad) } \\
3.75 E-10\end{array}$ \\
\hline \multicolumn{2}{|c|}{ Matching requirements } & Required & Result & \multicolumn{2}{|c|}{ Emittance Dilution Date } & & $\begin{array}{l}Q \\
\text { Variables }\end{array}$ & $\begin{array}{l}\text { G(T/m) } \\
\text { Design }\end{array}$ & $\begin{array}{c}\mathbf{G}(T / m) \\
\mathrm{Fil}\end{array}$ & $\mathbf{D G} / \mathbf{G}$ \\
\hline \multicolumn{11}{|l|}{ Case 1} \\
\hline (Fg. $10(a))$ & $\begin{array}{l}B x \\
A x\end{array}$ & $\begin{array}{r}139.0860 \\
0.2396\end{array}$ & $\begin{array}{r}139.10 C 4 \\
0.2395\end{array}$ & $\begin{array}{l}\text { (DBx/Bx)eq } \\
\text { (DBy/By)eq }\end{array}$ & $\begin{array}{l}0.0002 \\
0.0000\end{array}$ & & $\begin{array}{l}01 \\
02\end{array}$ & $\begin{array}{r}33.3290 \\
30.8610\end{array}$ & $\begin{array}{r}-33.6880 \\
30.8610\end{array}$ & $\begin{array}{l}0.0000 \\
0.0000\end{array}$ \\
\hline $0.3 \mathrm{~m}$ eta & $G x$ & 0.0076 & 0.0076 & & & & $\mathbf{Q 3}$ & 30.8810 & 30.8610 & 0.0000 \\
\hline At HEB alde & Bv & 154.8894 & 154.8859 & & & & 04 & .28 .3865 & -28.23865 & 0.0000 \\
\hline Im ETA & Ay & -0.3000 & -0.3000 & (DEta)loqu & 1.9315 & & 05 & 25.2355 & 25.2355 & 0.0000 \\
\hline \multirow[t]{5}{*}{ At Collider } & Gy & 0.0070 & 0.0070 & Sigm $\infty 0$ & 0.0001 & & Q8 & 32.9120 & .32 .9120 & 0.0000 \\
\hline & Etar & 1.0000 & -0.2500 & $O F(A, B) x$ & 0.0000 & & 07 & 33.8275 & 33.8275 & 0.0000 \\
\hline & Etay' & 0.0000 & 0.0000 & $D F(A, B) Y$ & 0.0000 & & 08 & -31.4875 & 31.4875 & 0.0000 \\
\hline & & & & DF(ETA)Y & 0.3211 & & Q9 & 32.6365 & 32.6265 & 0.0000 \\
\hline & & & & Y DF & 0.3211 & & Q10 & $-2 \pi . \pi 780$ & -27.7760 & 0.0000 \\
\hline \multicolumn{11}{|l|}{ Case 2 (nit) } \\
\hline \multirow{2}{*}{ (Fig. $10(b))$} & $8 x$ & 139.0360 & 139.0858 & $(08 \times / 8 x)=0$ & 0.0000 & & Q1 & .33 .6890 & 31.3130 & -0.0705 \\
\hline & $A x$ & 0.2398 & 0.2396 & (DBy/By)eq & 0.0000 & & 02 & 30.9610 & 30.5945 & -0.0096 \\
\hline $0.3 m$ eta & Gx & 0.0078 & 0.0076 & & & & 03 & 30.8610 & 30.5945 & -0.0088 \\
\hline $\begin{array}{l}\text { At HEB side } \\
\text { Im ETA }\end{array}$ & $\begin{array}{l}\text { By } \\
\text { Ay }\end{array}$ & $\begin{array}{c}154.8894 \\
-0.3000\end{array}$ & $\begin{array}{c}154.8897 \\
-0.3000\end{array}$ & (DEta) oqu & 0.0000 & & $\begin{array}{l}\text { Q4 } \\
05\end{array}$ & $\begin{array}{r}-29.9385 \\
25.2355\end{array}$ & $\begin{array}{r}-25.2545 \\
25.6985\end{array}$ & $\begin{array}{c}-0.0343 \\
0.0183\end{array}$ \\
\hline \multirow[t]{5}{*}{ At Collider } & Gy & 0.0070 & 0.0070 & Sigm ap & 0.0001 & & Q6 & 32.9120 & 34.3975 & 0.0631 \\
\hline & Etay & 1.0000 & 1.0000 & $D F(A, B) X$ & 0.0000 & & Q7 & 33.8275 & 33.4670 & -0.0107 \\
\hline & Etay & 0.0000 & 0.0000 & $D F(A, B) y$ & 0.0000 & & 08 & $-31 . .4875$ & 35.3535 & 0.1391 \\
\hline & & & & DF(ETA)Y & 0.0000 & & 09 & 32.6365 & 33.1025 & 0.0143 \\
\hline & & & & Y OF & 0.0000 & & Q10 & .27 .7760 & -27.7750 & 0.0000 \\
\hline \multicolumn{11}{|l|}{ Caso 3} \\
\hline$(\mathrm{Fg} \cdot 10(\mathrm{c}))$ & $8 x$ & 208.6290 & 68.6109 & $(D B \times / B \times) 00$ & 2.1237 & & Q1 & 23.6390 & -33.6990 & 0.0000 \\
\hline $50 \% B x, 50 \%$ By. & $A x$ & 0.2396 & 0.2350 & (DBy/By)eq & 2.0449 & & Q2 & 30.8610 & 30.8610 & 0.0000 \\
\hline $0.3 m \times a$ & Gx & 0.0051 & 0.0154 & & & & Q3 & 30.8610 & 30.8810 & 0.0000 \\
\hline At HEB side & BV & 232.3341 & 79.5216 & & & & Q4 & .29 .3965 & -29.9865 & 0.0000 \\
\hline$+50 \% 8 x_{1}+50 \%$ By & Ay & -0.3000 & -0.2302 & (DEta)equ & 1.9315 & & Q5 & 25.2355 & 25.2355 & 0.0000 \\
\hline Im ETA & Gy & 0.0047 & 0.0137 & Sigm ap & 0.0001 & & 06 & 32.9120 & 32.9120 & 0.0000 \\
\hline \multirow[t]{4}{*}{ At Collider } & Etay & 1.0000 & -0.3500 & $D F(A, B) x$ & 0.7219 & & 07 & 33.8275 & 33.8275 & 0.0000 \\
\hline & Etay' & 0.0000 & 0.0000 & $D F(A, B) y$ & 0.6867 & & Q8 & $-31 .: 875$ & 31.4875 & 0.0000 \\
\hline & & & & OF(ETA)Y & 0.2141 & & Q9 & 32.6365 & 32.6365 & 0.0000 \\
\hline & & & & Y DF & 0.9008 & & Q10 & $.27 . \pi .20$ & -27.7760 & 0.0000 \\
\hline \multicolumn{11}{|l|}{ Case $4(\pi)$} \\
\hline (Flo $10(d))$ & $8 x$ & 208.6290 & 208.6290 & $(D B \times / B \times)=0$ & 0.0066 & & 01 & 33.5390 & 33.s5es & -0.0039 \\
\hline $50 \% \mathrm{Bx},-50 \%$ By & $A x$ & 0.2398 & 0.2462 & (DBy/By)oa & 0.0017 & & 92 & 30.8610 & 31.4470 & 0.0190 \\
\hline $0.3 \mathrm{~m}$ et & $G_{x}$ & 0.0051 & 0.0051 & & & & Q3 & 30.9610 & 31.4470 & 0.0190 \\
\hline At HEB side & By & 232.3341 & 232.3505 & & & & Q4 & .28 .0965 & -23.0195 & -0.2059 \\
\hline$+50 \% 8 x,+50 \% 8 y$ & Ay & -0.3000 & -0.2394 & (DEla)equ & 0.0000 & & 05 & 25.2355 & 27.3315 & 0.0831 \\
\hline Im ETA & Gy & 0.0047 & 0.0047 & Sigm ap & 0.0001 & & 06 & 32.9120 & $-30.7 \pm 85$ & -0.0657 \\
\hline \multirow[t]{4}{*}{ At Colllder } & Etay & 1.0000 & 1.0000 & $D F(A, B) x$ & 0.0000 & & 08 & 33.8275 & 38.7120 & 0.1444 \\
\hline & Etay' & 0.0000 & 0.0000 & $D F(A, B) y$ & 0.0000 & & 07 & $-31 . .875$ & -40.0500 & 0.2719 \\
\hline & & & & DF(ETAY & 0.0000 & & 09 & 32.6365 & 31.8735 & 0.0234 \\
\hline & & & & Y DF & 0.0000 & & 010 & .27 .7760 & 26.5890 & -0.0427 \\
\hline
\end{tabular}



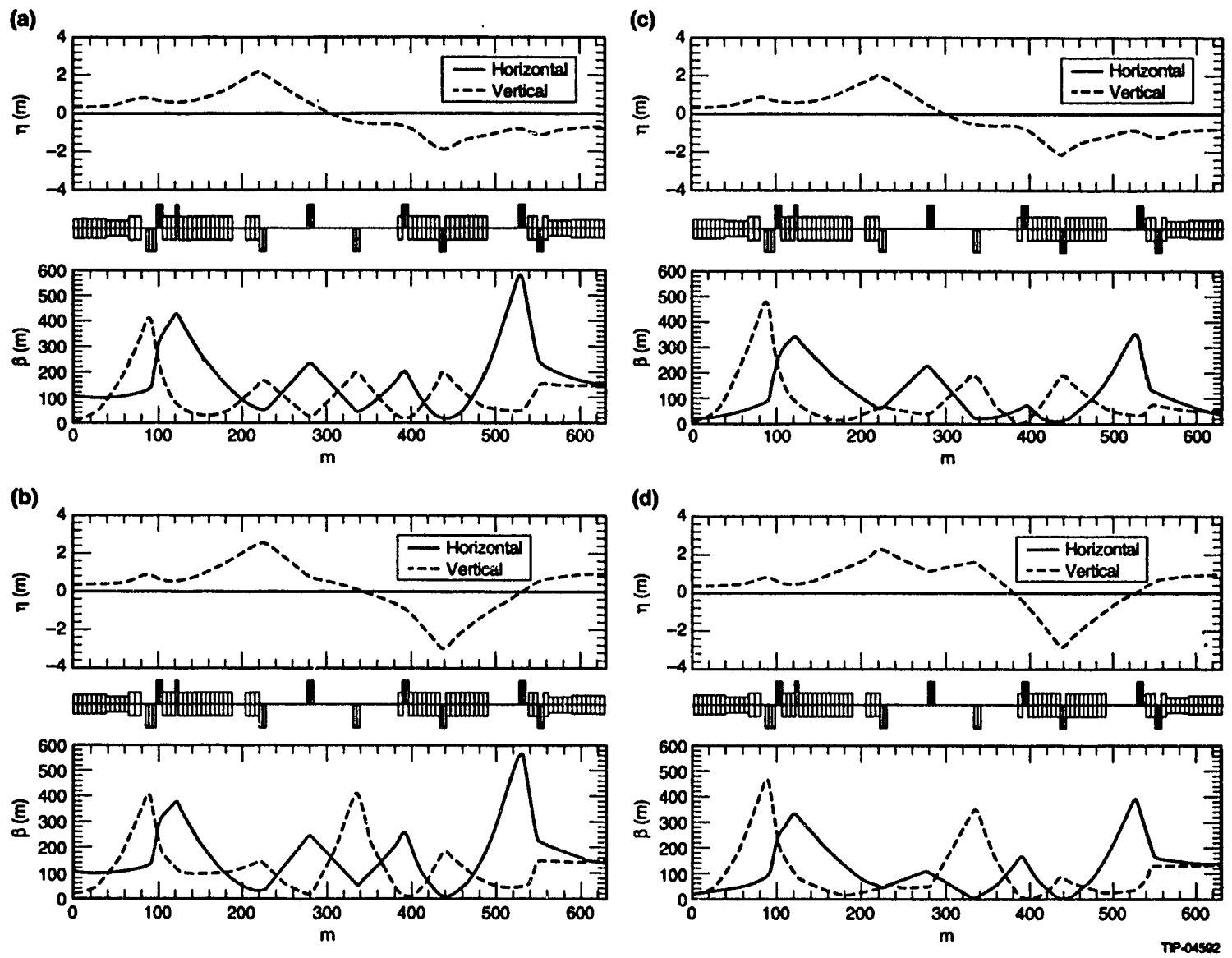

Figure 10. Optlcal Flexlbillty Study (Compact Resistive Lattice). (a) 0.3-m $\eta$ at HEB (no fitting).

(b) 0-3-m $\eta$ at HEB, 1-m at collider vary elght gradlents (Q1-Q9). (c) $0.3-m \eta$ and $-50 \% \beta$ at HEB. (d) $0.3-\mathrm{m} \eta$ and $-50 \% \beta$ at HEB matching to $1-\mathrm{m} \eta$ and $+50 \% \beta$ at Collider (Nine Gradlents varled: Q1-Q10). 


\section{REFERENCES}

1. Site-Specific Conceptual Design, SSCL-SR-1056, July 1990, p. 119-129.

2. F. Wang and R. Schailey, SSCL-ADOD-017B, March 1991; (revised Oct. 24, 1991, see HEB PDRR reports Part 3 April 2-3, 1992).

3. HEB PDRR Meeting Minutes, Action Item No. 8, April 20, 1992.

4. F. Wang, SSCL Internal Memo, "Issues of HEB-Collider Cold Solution," May 27, 1992.

5. F. Wang, SSCL Internal Memo, "HEB-Collider Hybrid Solution," July 22, 1992.

6. F. Wang, SSCL Collider Utility PDRR Part 4, January 27-28, 1993.

7. K. Brown, talk: "HEB-Collider Transport Line," September 15, 1992.

8. F. Wang, SSCL Internal Tech. Report, "HEB-Collider Transfer Major Dipole (Magnetic Design)," September 1991.

9. Karl L. Brown and Roger V. Servranckx, SLAC-PUB-3381, July 1984, p. 53-54.

10. M. J. Syphers, "Injection Mismatch and Phase Space Dilution," FN-458, June 1987.

11. J. McGill, Private communication, 1991.

12. F. Wang, SSCL Internal Memo, "EAC User's Guide," July 1991. 


\section{APPENDIX A}

\section{A.1 $\Delta \beta,(\Delta \beta / \beta)_{\text {eq }}$ and Emittance Dilution Factor $\Delta F$}

The intention here is to quantitatively link $\beta$ - or $\eta$-mismatch to emittance dilution. Starting point is M. Syphers's formula: ${ }^{10}$

$$
\Delta F=0.5\left[\left(\frac{\Delta \beta}{\beta}\right)_{e q} / \sqrt{\left(\frac{\Delta \beta}{\beta}\right)_{e q}+1}\right]^{2},
$$

where $\left(\frac{\Delta \beta}{\beta}\right)_{\text {.q }} \equiv D+\sqrt{D^{2}+1}$ and $D=0.5\left(\beta_{1} \gamma_{2}+\beta_{2} \gamma_{1}-2 \alpha_{1} \alpha_{2}\right)$.

For simplification, we consider only $\beta$-mismatch, keeping $\alpha$ the same and letting $k=\beta_{2} / \beta_{1}$. Then from Eq. (A.1):

$$
D=0.5\left(\left(k+\frac{1}{k}\right)\left(\alpha^{2}+1\right)-2 \alpha^{2}\right)
$$

So dilution should be a function of $\alpha$ and the ratio of $\beta_{2}$ and $\beta_{1}$ only.

At the collider side $\alpha_{y}=-0.3$ (WUSS lattice July 6, 1992).

When talking $+50 \% \beta_{y}$-mismatching, equivalents are $\Delta F=9 \%$ and $(\Delta \beta / \beta)_{e q}=0.53$, while with $-50 \% \beta_{\mathrm{y}}, \Delta F=27 \%$ and $(\Delta \beta / \beta)_{\text {eq }}=1.06$.

\section{A.2 $\Delta \eta, \Delta \eta_{\text {eq }}$, and Emittance Dilution}

$$
\Delta F_{\eta}=0.5\left(\left(\Delta \eta_{e q} \sigma_{p}\right) / \sigma_{0}\right)^{2}
$$

with $\Delta \eta_{\text {eq }} \equiv \sqrt{\Delta \eta^{2}+\left(\beta \Delta \eta^{\prime}+\alpha \Delta \eta\right)^{2}}$ and $\sigma_{0}=\sqrt{\varepsilon_{n} \beta /(\gamma \beta)}=\sqrt{\varepsilon \beta}$.

Again for simplification, assume different $\eta$ only $\left(\eta^{\prime}=0\right)$. Thus,

$$
\Delta F_{h}=0.5 \frac{\left(1+\alpha^{2}\right) \Delta \eta^{2} \sigma_{\mathrm{p}}^{2}}{\varepsilon \beta}
$$

Specified to the HEB beam: $\sigma_{p} \leq 1 \times 10^{-4}$ and $\varepsilon=3.75 \times 10^{-10} \mathrm{~m} \cdot \mathrm{rad},\left(\varepsilon_{\mathrm{n}}=0.8 \pi \mathrm{mm}-\mathrm{mrad}\right)$, then at the Collider side, $\beta_{y}=154.9 \mathrm{~m}, \alpha_{\mathrm{y}}=-0.3$, for $\Delta \eta=1 \mathrm{~m}: \Delta F=9.4 \%$, and at the HEB side, $\beta_{\mathrm{y}}=18.9 \mathrm{~m}$, $\alpha_{\mathrm{y}}=-0.03 \mathrm{~m}$, for $\Delta \eta=0.36 \mathrm{~m}: \Delta F=9.6 \%$.

\section{A.3 Thin-Lens Approximation for $\Delta F^{11}$}

From Eq. (A.1),

$$
\Delta F=D-1=\frac{1}{2}\left(\frac{\beta_{1}}{\beta_{2}}\left(1+\alpha_{2}^{2}\right)+\frac{\beta_{2}}{\beta_{1}}\left(1+\alpha_{1}^{2}\right)-2 \alpha_{1} \alpha_{2}\right)-1 .
$$


With thin-lens approximation, the quadrupole transfer matrix is

$$
M=\left[\begin{array}{cc}
1 & 0 \\
-\frac{1}{f} & 1
\end{array}\right]=\left[\begin{array}{cc}
1 & 0 \\
-\frac{G L}{\beta \varrho} & 1
\end{array}\right]
$$

Also across a thin lens, $\beta$ is unchanged, and Eq.(A.5) is reduced to

$$
\Delta \dot{r}=0.5\left(\alpha_{1}-\alpha_{2}\right)^{2} \text {. }
$$

Let $\alpha_{0}$ be the $\alpha$ value before this thin lens, and $\alpha_{1}$ the $\alpha$ value after the lens with design quadrupole gradient $G$ and $\alpha_{2}$ with gradient error $\Delta G$, by Eq. (A.6):

$$
\alpha_{2}=\frac{\beta}{f_{2}}+\alpha_{0} \text { and } \alpha_{1}=\frac{\beta}{f_{1}}+\alpha_{0}
$$

From Eq. (A.7), we obtain

$$
\Delta F=\frac{1}{2} \beta^{2}\left(\frac{1}{f_{1}}-\frac{1}{f_{2}}\right)^{2}=\frac{\beta^{2}}{2}\left(\frac{L G}{\beta \varrho}\right)^{2}\left(\frac{\Delta G}{G}\right)^{2} .
$$




\section{APPENDIX B:}

\section{SENSITIVITY TO QUADRUPOLE GRADIENT ERRORS}

One reason to keep small $\beta$-amplitude and weaker focussing-gradient strength in a lattice design is to make the matching less sensitive to quadrupole gradient errors. Quadrupole gradient errors of concern here are from current ripple or are reproducible for ramped power supplies that will have continuing effects on emittance dilution. Systematic calibration or setting errors will be eliminated by proper tuning.

The major effect of gradient errors is deterioration of $\beta$-matching. Adopting M. Syphers's formula and J. McGill's approximation (see Appendix A), the fractional emittance dilution due to $\beta$-mismatching is

$$
\frac{\Delta \varepsilon}{\varepsilon}=\sum_{i} \frac{1}{2}\left(\frac{G_{i} L_{i} \beta_{i}}{B \varrho}\right)^{2}(\Delta G / G)_{i}^{2}=F \cdot(\Delta G / G)_{r m s}^{2}
$$

where $\mathrm{BQ}$ is the particle magnetic rigidity, and $\Delta G / G$ is the fractional gradient error. Sum is over all quadrupoles. $F$, the dilution coefficient, is shown in Table B-1.

Table B-1 lists the coefficient terms of quadrupoles in each lattice, as well as the sum of them $(F)$, which is proportional to the emittance dilution when all quadrupole supplies have similar rms deviation value.

The two achromats lattices are more sensitive to gradient errors because they consist of more quadrupoles and have larger average $\beta$. The hybrid one has the minimum $F$. Largest value of $F$ is about 5 times greater than the smallest value of $F$ for these lattices.

The reliability of the above estimation is confirmed by comparing results with those from the error simulation program. ${ }^{12}$ Figure B-1 depicts the error sensitivity results for the compact resistive lattice directly from optics calculations with random gradient errors (normal distribution) assigned for each quadrupole. The parameters shown in the figures are $\Delta \beta x_{e q} / \beta x_{e q}$, the fractional change of equivalent $\beta$-amplitude at the end of the line, and the emittance dilution factor $\Delta F$, both defined in Eq. (B.1).

The results agree with Table B-1 by Eq. (B.1). For example, from Table B-1 with rms gradient error of 0.002 , emittance dilution is $0.127 \%$ for $X$ direction and $0.064 \%$ for $Y$ direction. Counting one $\sigma$-width trial number ( $\sim 68 \%$ of total trials) in Figures B-1(c) and B-1(d), we get nearly the same values as those. 
TABLE B-1. COMPARISON OF SENSITIVITY TO QUADRUPOLE GRADIENT ERRORS.

\begin{tabular}{|c|c|c|}
\hline NAME & UM) & GRADIENT(T/M) \\
\hline 00 & 1.6000 & -176.2580 \\
\hline QF & 1.6000 & 187.8745 \\
\hline$Q 0$ & 1.6000 & - \\
\hline QF & 1.6000 & 138.1155 \\
\hline$Q D$ & 1.6000 & -162.3040 \\
\hline 0.5 & 1.6000 & 122.1070 \\
\hline Q6 & 1.0500 & -96.5595 \\
\hline 07 & 1.0500 & 158.2915 \\
\hline Q8 & 1.0500 & $-i 50.3 \mathrm{eos}$ \\
\hline QD & 1.6000 & -18.2405 \\
\hline OF & 1.6000 & 138.1175 \\
\hline$Q D$ & 1.6000 & .138 .1175 \\
\hline QF & 1.6000 & 187.7380 \\
\hline$Q D$ & 1.6000 & -143.3045 \\
\hline
\end{tabular}

The Compact Resistive Solution

\begin{tabular}{|c|c|c|}
\hline QNAME & $L(M)$ & GRADIENTTT/M) \\
\hline Q1 & 6.0000 & .33 .6890 \\
\hline Q1 & $3.000 \mathrm{C}$ & -33.8820 \\
\hline Q2 & 6.0000 & 30.8610 \\
\hline 03 & 6.0000 & 30.8810 \\
\hline Q4 & 6.0000 & -28.9865 \\
\hline QSF & 6.0000 & 25.2355 \\
\hline Q60 & 6.0000 & -32.9120 \\
\hline Q7F & 6.0000 & 33.8275 \\
\hline Q8D & 6.0000 & -31.4875 \\
\hline 09 & 6.0000 & 32.6365 \\
\hline Q10 & 6.0000 & -27.7 .60 \\
\hline
\end{tabular}

The Hybrid Solution

$\begin{array}{llc}\text { NAME } & \text { LM) } & \text { GRADIENT(T/M) } \\ \text { Q1S } & 1.6000 & \cdot i 58 . i 425 \\ \text { Q2 } & 6.0000 & 24.1795 \\ \text { Q3 } & 3.0000 & 18.1915 \\ \text { Q4 } & 3.0000 & 13.0045 \\ \text { Q5 } & 6.0000 & -24.8420 \\ \text { Q6F } & 6.0000 & 26.3340 \\ \text { QD } & 6.0000 & -27.6690 \\ \text { QF } & 6.0000 & 27.8690 \\ \text { Q70 } & 6.0000 & -25.1445 \\ \text { Q8 } & 3.0000 & -5.6835 \\ \text { Q9 } & 3.0000 & .1 .5205 \\ \text { Q10 } & 3.0000 & 30.0980\end{array}$

$\begin{array}{llr}\text { Q10 } & 3.0000 & 30.0980 \\ \text { Q115 } & 1.0500 & -140.3525\end{array}$

The Old Resistive Solution

$\begin{array}{lrc}\text { NAME } & \text { LIM) } & \text { GRADIENT(T/M) } \\ \text { Q1 } & 11.0000 & -28.3865 \\ Q 2 & 11.0000 & 26.4910 \\ Q 3 & 5.5000 & -7.4195 \\ Q 3 & 5.5000 & .29 .6530 \\ Q 4 & 11.0000 & 22.4820 \\ Q 5 & 5.5000 & -27.2695 \\ Q 8 & 5.5000 & 28.6415 \\ Q 7 & 5.5000 & -25.9490 \\ Q 7 & 5.5000 & 9.0260 \\ Q 8 & 5.5000 & 24.2050 \\ Q 9 & 11.0000 & -23.3505 \\ Q 10 & 5.5000 & 29.3165 \\ Q 11 & 5.5000 & .29 .2310\end{array}$

\begin{tabular}{rr} 
BETA-X & \multicolumn{1}{l}{ BETA-Y } \\
116.1727 & 268.7331 \\
489.0868 & 33.4484 \\
12.5198 & 327.3668 \\
259.0540 & 90.4900 \\
113.3782 & 288.1344 \\
255.0958 & 83.4002 \\
55.4594 & 87.4909 \\
73.9307 & 152.6714 \\
34.7360 & 493.4178 \\
51.8142 & 432.5595 \\
233.9020 & 151.7668 \\
10.1669 & 522.2899 \\
176.5109 & 11.5847 \\
53.5791 & 429.0045
\end{tabular}

BETA-X BETA-Y

$137.9200 \quad 414.0408$

$206.8468 \quad 320.4125$

$310.1233 \quad 205.6656$

$424.0952 \quad 89.0480$

$51.2336 \quad 156.0768$

$235.6479 \quad 30.1484$

$48.4083 \quad 201.0228$

$201.7063 \quad 18.8370$

$15.7372 \quad 193.4612$

$578.8738 \quad 46.8780$

$237.9227 \quad 147.7405$

SUM $F=$

BETA-X BETA-Y

$124.1087 \quad 281.6793$

$360.8029 \quad 89.9257$

$384.9854 \quad 52.8652$

$210.2483 \quad 40.7834$

$24.2395 \quad 297.3577$

$314.1067 \quad 36.9311$

$58.9451 \quad 151.9414$

$149.7672 \quad 36.3443$

$29.9737 \quad 182.6077$

$64.6036 \quad 134.6716$

$131.1204 \quad 96.7556$

$448.1577 \quad 67.7391$

218.3808

149.9916

BETA-X BETA-Y

$161.3399 \quad 458.8160$

$506.3062 \quad 99.1843$

$90.8802 \quad 62.1883$

$26.4560 \quad 367.1841$

357.2793 109.0587

$101.6415 \quad 235.2640$

$62.9353 \quad 225.1731$

$20.6711 \quad 291.0493$

242.1876 103.2969

404.994: $\quad 124.2181$

$153.5710 \quad 338.4647$

$437.6811 \quad 5.7477$

56.8344
1/2RBXGBR) $2 \quad 1 / 2(L B y G / B R)^{2}$

$\begin{array}{rr}12.0739 & 64.0075 \\ 242.6468 & 1.1349 \\ 0.0861 & 58.8771 \\ 36.8687 & 4.4986 \\ 9.7524 & 54.5451 \\ 27.3435 & 1.7281 \\ 0.3557 & 0.8852 \\ 1.6986 & 7.2437 \\ 0.3383 & 68.2697 \\ 0.0257 & 1.7929 \\ 30.0578 & 12.6545 \\ 0.0568 & 149.8695 \\ 31.6250 & 0.1362 \\ 1.6979 & 108.8519 \\ 395.23 & 535.09\end{array}$

$1 / 2(L B \times G / B R)^{2} \quad 1 / 2(L B Y G / B R)^{2}$

$\begin{array}{rr}6.7430 & 78.7937 \\ 4.9164 & 11.7968 \\ 37.0974 & 16.3154 \\ 69.3748 & 1.8390 \\ 0.8932 & 9.3857 \\ 14.3221 & 0.2344 \\ 0.9448 & 17.7278 \\ 18.8553 & 0.1644 \\ 0.0994 & 15.0286 \\ 44.5540 & 0.9480 \\ 17.6875 & 6.8201 \\ 317.49 & 159.05\end{array}$

$1 / 2(\mathrm{~B} \times G / B R)^{2} \quad 1 / 2(L B y G / B R)^{2}$

11.0942

30.8241

4.9662

0.7569

0.1469

27.7104

1.0773

6.9547

0.1949

0.0137

0.0040

18.4218

11.7352

113.90

1/2(LBXGBA $)^{2}$

28.5123

1/2(LBYG/BR) 2

244.8344

0.1547

0.2094

87.8261

2.6144

1.1058

0.0979

1.6262

32.7028

17.5045

56.0296

0.9395

SUM Fz
57.1478

1.9148

0.0936

0.0285

22.0997

0.3831

7.1580

0.4096

72342

0.0593

0.0022

0.4209

5.5362

102.49

230.5826

9.3977

0.0725

40.3444

8.1833

14.0069

14.1548

19.4112

0.2958

3.0765

35.0270

0.0097

70.0929

494.66 
(a)

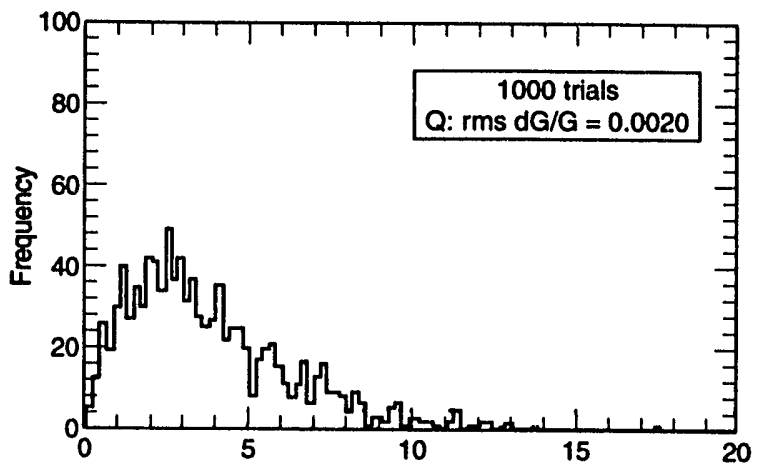

(b)

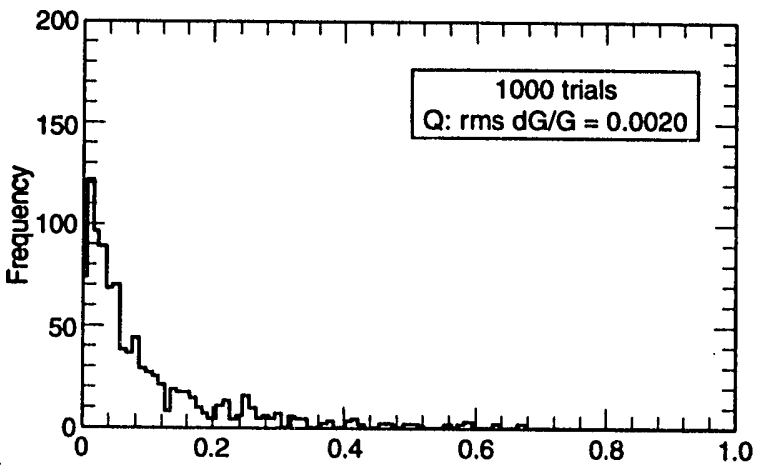

(c)

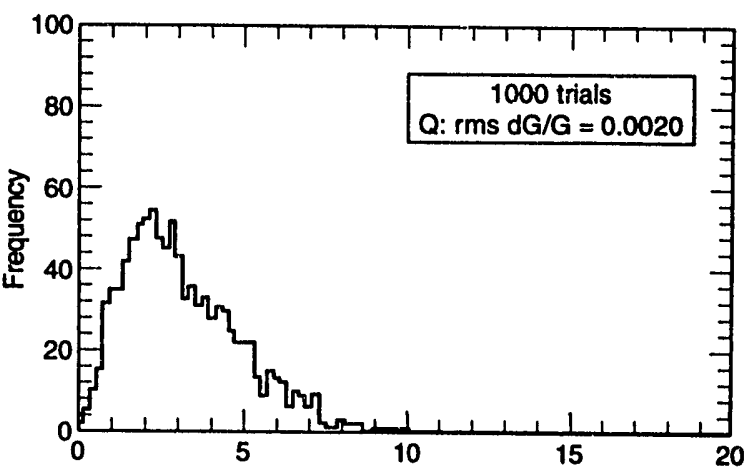

(d)

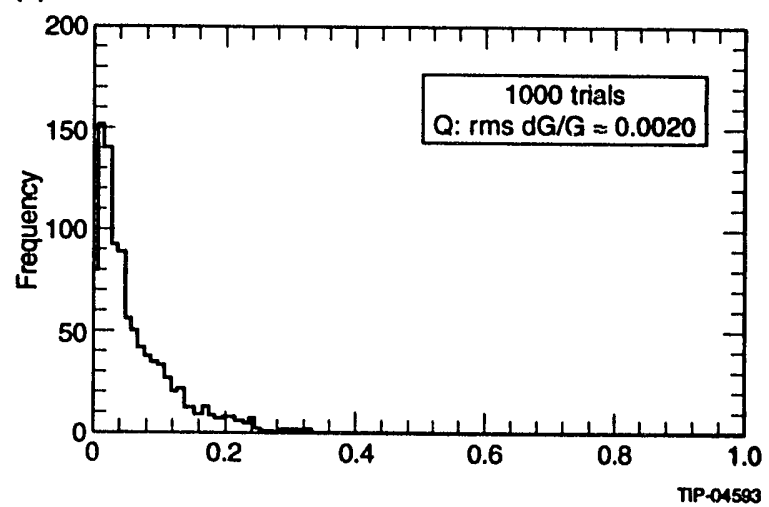

Figure B-1. Sensitivity to Gradient Errors (Compact Resistive Lattice). (a) d $\beta x e q / \beta x e q \%$. (b) \% Emittance

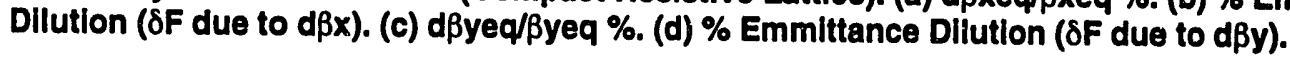



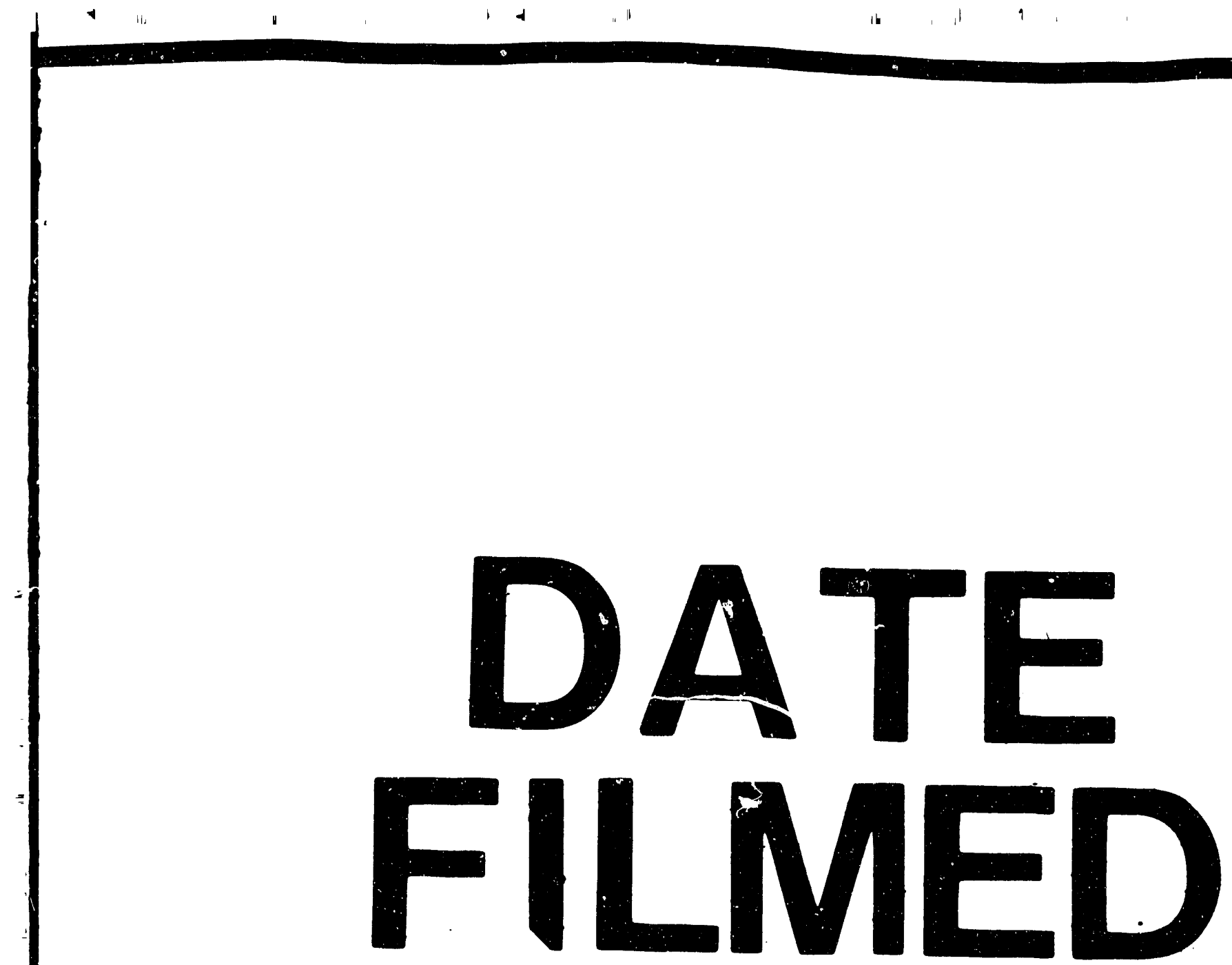

9

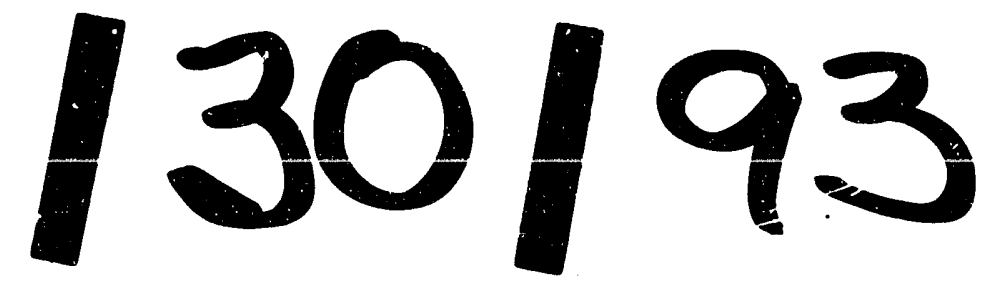




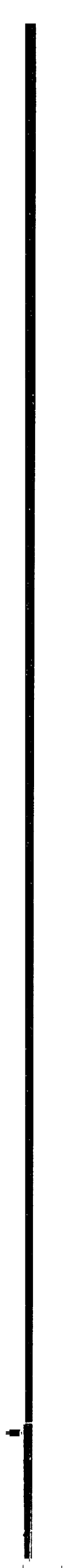

